\title{
Colourful Poincaré symmetry, gravity and particle actions
}

\author{
Joaquim Gomis, ${ }^{a}$ Euihun Joung, ${ }^{b}$ Axel Kleinschmidt ${ }^{c, d}$ and Karapet Mkrtchyan ${ }^{e}$ \\ ${ }^{a}$ Departament de Física Quàntica $i$ Astrofísica \\ and Institut de Ciències del Cosmos (ICCUB), \\ Universitat de Barcelona, \\ Martí i Franquès, ES-08028 Barcelona, Spain \\ ${ }^{b}$ Department of Physics, Kyung Hee University, \\ Seoul 02447, Korea \\ ${ }^{c}$ Max-Planck-Institut für Gravitationsphysik (Albert-Einstein-Institut), \\ Am Mühlenberg 1, DE-14476 Potsdam, Germany \\ ${ }^{d}$ International Solvay Institutes, \\ ULB-Campus Plaine CP231, BE-1050 Brussels, Belgium \\ e Theoretical Physics Group, Blackett Laboratory, \\ Imperial College London SWr 2AZ, U.K. \\ E-mail: joaquim.gomis@ub.edu, euihun.joung@khu.ac.kr, \\ axel.kleinschmidt@aei.mpg.de, k.mkrtchyan@imperial.ac.uk
}

ABSTRACT: We construct a generalisation of the three-dimensional Poincaré algebra that also includes a colour symmetry factor. This algebra can be used to define coloured Poincaré gravity in three space-time dimensions as well as to study generalisations of massive and massless free particle models. We present various such generalised particle models that differ in which orbits of the coloured Poincaré symmetry are described. Our approach can be seen as a stepping stone towards the description of particles interacting with a nonabelian background field or as a starting point for a worldline formulation of an associated quantum field theory.

KEYwords: Global Symmetries, Space-Time Symmetries

ArXiv ePrint: 2105.01686 


\section{Contents}

1 Introduction 1

2 Colouring the Poincaré algebra in 3 space-time dimensions 3

3 Coloured gravity in 3d Minkowski space-time $\quad 8$

3.1 Invariant bilinear form 8

$\begin{array}{lll}3.2 & \text { Coloured gravity action in 3d Minkowski space } & 10\end{array}$

4 Free coloured particle model $\quad 12$

$\begin{array}{lll}4.1 \text { Coloured Minkowski space } & 12\end{array}$

$\begin{array}{lll}4.2 & \text { Different types of massive particle actions } & 14\end{array}$

4.2.1 Uncoloured Minkowski space 14

$\begin{array}{ll}\text { 4.2.2 Coloured Minkowski space } & 16\end{array}$

$\begin{array}{lll}4.3 \text { Coadjoint orbits of } \mathfrak{s u}(N, N) & 19\end{array}$

$\begin{array}{lll}\text { 4.3.1 Massive particles: semi-simple orbits } & 19\end{array}$

4.3.2 Massless particles: nilpotent orbits 21

4.4 Massive coloured particle in component form 22

\begin{tabular}{ll} 
4.5 Constraint structure and degrees of freedom 25 \\
\hline
\end{tabular}

$\begin{array}{lll}4.6 & \text { Examples for small } N & 27\end{array}$

$\begin{array}{lll}4.7 & \text { Massless coloured particle } & 29\end{array}$

4.8 Reductions to subspaces 31

5 Free coloured particle in AdS background 32

5.1 Uncoloured particle in AdS 33

5.2 Coloured particle in AdS 34

6 Conclusions $\quad 35$

$\begin{array}{ll}\text { A Derivation from embedding in AdS algebra } & 39\end{array}$

$\begin{array}{ll}\text { B Free massive Poincaré particle } & 40\end{array}$

C General remarks on particle actions 44

D From the coloured Hamiltonian to the Lagrangian action 46 


\section{Introduction}

Space-time symmetries that are larger than those realised in conventional gravitational systems, including bosonic generators in non-trivial representations of isometry algebra are usually ruled out in field theories of finitely many interacting particles. The ColemanMandula theorem establishes that such symmetries are not compatible with a non-trivial S-matrix [1]. However, there are ways to evade the Coleman-Mandula theorem.

Einstein gravity in three dimensions can be written in Chern-Simons form [2,3]. This formulation is based on gauging the global symmetry algebra and is background independent as well as directly related to the Einstein-Hilbert formulation of gravity, if the dreibein is non degenerate. Such a formulation can be generalised to higher-spin gravities without matter [4-6] thus providing consistent examples of gravitational theories with extended symmetries. These theories lack bulk propagating degrees of freedom and in this way avoid no-go theorems based on S-matrix considerations. Other possible extensions that have been considered are given by Chern-Simons actions based on relativistic or non-relativistic Maxwell algebra extensions of the Poincaré algebra, see for example [7-12].

A different extension of three-dimensional space-time symmetries is realised in coloured gravity [13]. There, instead of adding generators corresponding to massless fields of spin $s \geq 3$, one has a colour extension of the isometry itself, with multiple copies of generators which correspond to Killing vectors and scalars associated to massless spin-two and spinone fields. Such an extension is non-trivial for associative algebras of isometries $[13,14]$, the first example was provided in [15]. For the Poincaré algebra in arbitrary dimensions, such a colouring requires the colour algebra to be commutative and associative [16-18], and together with the requirement of positive-definite bilinear form for the colour algebra (needed for unitarity) is trivial as shown in [19]: the corresponding multi-gravity is described by a sum of mutually non-interacting Einstein-Hilbert actions. ${ }^{1}$ In three dimensions, the (A)dS $\mathrm{d}_{3}$ isometry algebra, being a real form of $\mathfrak{s l}_{2} \oplus \mathfrak{s l}_{2}$, can be extended to an associative algebra by a double central extension. Incidentally, the same central extension allows taking different non-relativistic limits in three dimensions [21].

It is a generic problem to couple matter to the gravitational systems with extended symmetries such as the coloured (higher-spin) gravity given by a Chern-Simons action in three dimensions. Lagrangian formulations for known examples of higher-spin gravities with matter in three dimensions $[22,23]$ are not available yet, ${ }^{2}$ despite some steps in that direction (see [26] and references therein). In this paper we take a step back and instead try to understand it at the level of the worldline formulation of particles, since it is the first step towards an associated quantum field theory from a worldline approach.

We first note that in theories with extended space-time symmetries the very notion of particle has to be reconsidered. Since particles in field theories as we know them are

\footnotetext{
${ }^{1}$ In three space-time dimensions one can also deform the Poincaré algebra which gives a theory different from Einstein gravity, see [20] where also an extension to a collection of spin-two fields governed by this deformed algebra was studied.

${ }^{2}$ In [24] an interesting proposal was made using a higher-dimensional space. See also [25] for a similar idea.
} 
defined as (unitary) irreducible representations of the isometry algebra, for extended spacetime symmetry algebras, one should consider the irreducible representations of the extended algebra. In principle, the representations of the larger symmetry algebra should decompose in terms of the representations of the original isometry algebra. We just note here, that in case that the representation of the larger algebra has higher Gelfand-Kirillov (GK) dimension (see e.g. [27] for the definition) than that of regular particles, which will be true for the cases of our interest, the corresponding spectrum should be expected to contain infinite number of particles.

A natural expectation is that systems with extended symmetries may allow for a mechanism of (spontaneous) symmetry breaking, that would lead to a regime with a more conventional gravitational system and massive particles coupled to it, much like in string theory. If such a scenario is realistic, then the notion of space and time is emergent and makes sense only for low-energy systems while at high energies (beyond the Planck scale?) it has to be abandoned or replaced by a more general notion. On the other hand, the higher symmetries of gravitational systems and their representations can be studied independently of the possible space-time interpretations.

We will show in this paper that not only the $(\mathrm{A}) \mathrm{dS}_{3}$ but also the Poincaré algebra in three dimensions can be extended via a colour decoration. This example of the coloured Poincaré symmetry is interesting for several reasons.

In Minkowski space the motion of a relativistic particle in a fixed electro-magnetic field is described by the Lorentz force. In the case of constant electro-magnetic field the symmetry algebra is given by the Bacry-Combe-Richards algebra [28] that contains four spacetime translations that do not commute, two Lorentz boost transformations and two central charges. Another possibility is to enlarge the Poincaré algebra with tensorial non-central charges, leading to the so-called Maxwell algebra [29]. If one also enlarges Minkowski space with tensorial bosonic coordinates, the particle moving in a constant electro-magnetic field can be made invariant under the Maxwell symmetry [30]. In an even further enlarged space-time one can generalise the Maxwell algebra to a free Maxwell algebra [31] that can describe the motion of the particle in a general electromagnetic field.

The motion of a relativistic particle with colour-flavour indices in a fixed Yang-Mills background, with space-time coordinates $x^{a}$ and colour coordinates $y_{I}$ (in the adjoint of $\mathrm{SU}(N)$ ), was first studied by Wong [32]. As in the electro-magnetic case it will be interesting to see whether the motion of a coloured particle in a fixed Yang-Mills background has additional invariances associated with the colour coordinates, in particular, whether there exists a non-abelian generalisation of the Maxwell algebra for the case of covariantly constant Yang-Mills background [33, 34].

In this paper we take some first steps in this direction by constructing the coloured $(\mathfrak{s}) \mathfrak{u}(N)$ Poincaré algebra in three dimensions. ${ }^{3}$ The generators are generalised commuting translations, internal symmetry transformations and coloured Lorentz transformations. There is a natural generalisation of Minkowski space to a coloured Minkowski space with coordinates $\left(x^{a}, y_{I}, x_{I}^{a}\right)$ where $x^{a}$ are the ordinary space-time coordinates, $y_{I}$ are the colour

\footnotetext{
${ }^{3}$ We comment on generalisations to higher dimensions in the conclusions.
} 
internal coordinates and $x_{I}^{a}$ are coloured space-time coordinates. There is a coloured generalisation of the Poincaré transformations in this space. Since the space-time is enlarged, the usual Coleman-Mandula theorem no longer applies directly. Interestingly, one can restrict to the subspace defined by $x_{I}^{a}=0$ at the expense of breaking the full coloured Poincaré symmetry to Poincaré and colour transformations. This subspace has the same coordinates as the Wong particle.

In the coloured Minkowski space we construct massive and massless particle actions that are invariant under the coloured generalisation of the Poincaré algebra using a variety of methods. The methods differ in which coadjoint orbits of the coloured Poincaré algebra we consider and we discuss their classification in some detail. ${ }^{4}$ The choice of orbit is at the heart of Wigner's construction of induced representations and presents the starting point for a particle interpretation. If we restrict our constructions to the subspace $x_{I}^{a}=0$ we can recover the free Wong equations.

We also construct the particle action with a coloured $\mathrm{AdS}_{3}$ symmetry $[13,14]$, in the flat limit we recover the coloured Poincaré particle found before. We also notice that subalgebra $\mathfrak{u}(N, N)$ of coloured Poincaré, in three dimensions, with an appropriate identification of the generators of coloured two-dimensional translations gives the coloured $\mathrm{AdS}_{2}$ algebra. This algebra could be used to construct the coloured $\mathrm{AdS}_{2}$ particle or the one-dimensional coloured conformal particle mechanics that generalises to the coloured case the one of reference [36].

The organisation of the paper is as follows. We first discuss the algebraic method for colouring the three-dimensional Poincare algebra in section 2. In section 3, we show that there is an invariant bilinear form on the $N$-coloured Poincare algebra cPoin $3(N)$ and use it to construct a Chern-Simons theory corresponding to coloured Poincaré gravity. Section 4 discusses particle actions built from $\operatorname{cPoin}_{3}(N)$ that utilise coloured Minkowski that is also introduced there. Various types of particle actions differ by the co-adjoint orbits of $\operatorname{cPoin}_{3}(N)$ they describe. We also consider the coloured particle in an AdS background in section 5 before offering some concluding remarks in section 6 . Several appendices contain complementary details.

\section{Colouring the Poincaré algebra in 3 space-time dimensions}

At the kinematic level, we first address the problem of adding colour indices to the Poincaré algebra, such that the new generators form a Lie algebra. Since the tensor product of two Lie algebras is not a Lie algebra, we have to resort to a different construction. In $D=3$ space-time dimensions a similar problem has been studied in the case of AdS and higher spin algebras $[13,14]$ that we rely on. The construction is based on embedding the Poincaré Lie algebra into an associative algebra and then tensoring it with an associative 'colour' algebra. Since the tensor products of associative algebras is associative and every associative algebra can be turned into a Lie algebra using the commutator, the construction yields a Lie algebra that contains a colouring of the original Poincaré Lie algebra.

\footnotetext{
${ }^{4}$ For a discussion of coadjoint orbits in the uncoloured case see for example [35].
} 
Before addressing the embedding the lifting of the Poincare algebra to an associative algebra, we first discuss the colour algebra. This we take to be $\mathfrak{g l}(N, \mathbb{C})$ with $\mathfrak{u}(N)$ as its basis over $\mathbb{C}$, corresponding to the subspace of all anti-hermitian $(N \times N)$ matrices. The product in this basis is given by (see, e.g., [37])

$$
T^{I} T^{J}=-\frac{1}{N} \delta^{I J} \mathbf{I}+\frac{1}{2}\left(\mathrm{i} d_{K}^{I J}+f_{K}^{I J}\right) T^{K}, \quad \mathbf{I} T^{I}=T^{I} \mathbf{I}=T^{I}, \quad \mathbf{I} \mathbf{I}=\mathbf{I} .
$$

Here, $T^{I}$ are the traceless generators of $\mathfrak{s u}(N)$ with $I=1, \ldots, N^{2}-1$ and $\mathbf{I}$ is the $N \times N$ identity matrix. The structure constants $f^{I J}{ }_{K}$ in $\left[T^{I}, T^{J}\right]=f^{I J}{ }_{K} T^{K}$ are real while the real $d^{I J}{ }_{K}$ are the invariant tensors mapping the symmetric product of two $\mathfrak{s u}(N)$ adjoint representations back to the adjoint of $\mathfrak{s u}(N)$. They exist for $N>2$ and the identities satisfied by the invariant tensors turn $\mathfrak{g l}(N, \mathbb{C})$ into an associative algebra. Here, $\delta^{I J}$ is the invariant metric on the adjoint of $\mathfrak{s u}(N)$. When using this to raise indices, $d^{I J K}$ becomes totally symmetric and $f^{I J K}$ becomes totally antisymmetric. When colouring the Poincaré algebra later, we shall use only a real slice $\mathfrak{u}(N) \subset \mathfrak{g l}(N, \mathbb{C})$, and for this reason we shall refer the associative algebra $\mathfrak{g l}(N, \mathbb{C})$ as $\mathfrak{u}(N)$ with an abuse of notation.

To write the algebra in a uniform way, we denote the anti-hermitian element $T^{0}=\mathrm{i} \mathbf{I}$ and use the indices $\widehat{I}=0,1, \ldots, N^{2}-1$ to label all the generators of $\mathfrak{u}(N)$. The associative product is given by

$$
T^{\widehat{I}} T^{\widehat{J}}=\frac{1}{2} \widehat{f} \widehat{I J} \widehat{K} T^{\widehat{K}}+\frac{\mathrm{i}}{2} \widehat{d}^{\widehat{I J}} \widehat{K} T^{\widehat{K}}
$$

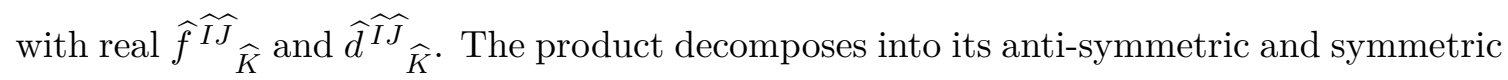
parts according to

$$
\left[T^{\widehat{I}}, T^{\widehat{J}}\right]=\widehat{f}^{\widehat{I J}} \widehat{K}^{\widehat{K}}, \quad\left\{T^{\widehat{I}}, T^{\widehat{J}}\right\}=\mathrm{i} \widehat{d}^{\widehat{I J}} \widehat{K} T^{\widehat{K}} .
$$

The tensors $\widehat{f}^{\widehat{I J}} \widehat{K}$ and $\widehat{d}^{\widehat{I J}} \widehat{K}$ satisfy identities analogous to the usual $\mathfrak{s u}(N)$ identities. When splitting the adjoint indices they have components

$$
\begin{array}{lll}
\widehat{f}^{0 \widehat{I}_{\widehat{K}}}=0, & \widehat{f}^{I J}=0, & \widehat{f}^{I J}{ }_{K}=f^{I J}{ }_{K}, \\
\widehat{d}^{0 \widehat{I}_{\widehat{K}}}=2 \delta_{\widehat{K}}^{\widehat{I}}, & \widehat{d}^{I J}{ }_{0}=\frac{2}{N} \delta^{I J}, & \widehat{d}^{I J}{ }_{K}=d^{I J}{ }_{K} .
\end{array}
$$

The Poincaré Lie algebra in $(2+1)$ space-time dimensions has Lorentz and translations generators $M_{a}, P_{a}$ and commutation relations ${ }^{5}$

$$
\left[M_{a}, M_{b}\right]=\varepsilon_{a b}^{c} M_{c}, \quad\left[M_{a}, P_{b}\right]=\varepsilon_{a b}^{c} P_{c}, \quad\left[P_{a}, P_{b}\right]=0 .
$$

Its embedding into an associative algebra can be achieved as follows. The starting point is to write the generators of the Poincaré algebra as tensor products

$$
M_{a}=L_{a} \otimes \mathcal{I}, \quad P_{a}=L_{a} \otimes \mathcal{J},
$$

\footnotetext{
${ }^{5}$ The indices $a, b, c=0,1,2$ are Minkowski space-time indices. We use $\varepsilon^{012}=+1$ and indices are raised and lowered with the $(-++)$ Minkowski metric.
} 
where $\mathcal{I}$ and $\mathcal{J}$ are generators of an associative abelian algebra $\mathfrak{a}$ (over $\mathbb{R}$ ) satisfying

$$
\mathcal{I}^{2}=\mathcal{I}, \quad \mathcal{J}^{2}=0, \quad \mathcal{I} \mathcal{J}=\mathcal{J} \mathcal{I}=\mathcal{J}
$$

while $L_{a}$ are generators of $\mathfrak{s l}(2, \mathbb{R}) \cong \mathfrak{s u}(1,1)$ satisfying $\left[L_{a}, L_{b}\right]=\varepsilon_{a b}{ }^{c} L_{c}$. For reality of the $\mathfrak{s l}(2, \mathbb{R})$ structure constants $\varepsilon^{a b c}$ we require $L_{a}^{\dagger}=-L_{a}$. For the anti-hermiticity of $M_{a}$ and $P_{a}$, we assign to $\mathcal{I}$ and $\mathcal{J}$ the hermiticity properties ${ }^{6}$

$$
\mathcal{I}^{\dagger}=\mathcal{I}, \quad \mathcal{J}^{\dagger}=\mathcal{J}
$$

Even though $\mathfrak{s l}(2, \mathbb{R})$ is only a Lie algebra and not associative, the Lie bracket $[x \otimes$ $a, y \otimes b]=\frac{1}{2}([x, y] \otimes\{a, b\}+\{x, y\} \otimes[a, b])=[x, y] \otimes a b$ on $\mathfrak{s l}(2, \mathbb{R}) \otimes \mathfrak{a}$ is well-defined since $\mathfrak{a}$ is abelian, a fact that was also used in the context of (conformal) gravity [18, 39]. The Lie algebra $\mathfrak{s l}(2, \mathbb{R}) \otimes \mathfrak{a}$ can now be embedded in an associative algebra by extending the first factor and defining the eight-dimensional associative algebra

$$
\mathfrak{A}=\mathfrak{u}(1,1) \otimes \mathfrak{a}=\left\langle L_{a} \otimes \mathcal{I}, L_{a} \otimes \mathcal{J}, \mathrm{i} \mathbf{I}_{2} \otimes \mathcal{I}, \mathrm{i} \mathbf{I}_{2} \otimes \mathcal{J}\right\rangle
$$

Here, the associative product on $\mathfrak{u}(1,1)$ is given by

$$
L_{a} L_{b}=\frac{1}{2} \varepsilon_{a b}^{c} L_{c}+\frac{1}{4} \eta_{a b} \mathbf{I}_{2}
$$

with $\mathbf{I}_{2}$ being the identity element of the algebra, with $\mathbf{I}_{2}^{\dagger}=\mathbf{I}_{2}$. This formula is similar to $(2.1)$.

The coloured Poincaré algebra in $D=3, \operatorname{cPoin}_{3}(N)$, is then defined to be the Lie algebra associated with the associative algebra given by the tensor product

$$
\operatorname{cPoin}_{3}(N)=\mathfrak{A} \otimes \mathfrak{u}(N) .
$$

A basis of the vector space $\mathrm{cPoin}_{3}$ is given by the generators

$$
\begin{aligned}
M_{a}^{\widehat{I}} & =-\mathrm{i} L_{a} \otimes \mathcal{I} \otimes T^{\widehat{I}}, \\
P_{a}^{\widehat{I}} & =-\mathrm{i} L_{a} \otimes \mathcal{J} \otimes T^{\widehat{I}}, \\
N^{\widehat{I}} & =\mathbf{I}_{2} \otimes \mathcal{I} \otimes T^{\widehat{I}}, \\
Q^{\widehat{I}} & =\mathbf{I}_{2} \otimes \mathcal{J} \otimes T^{\widehat{I}} .
\end{aligned}
$$

The factors of $\mathrm{i}$ are introduced such that all generators are anti-hermitian.

\footnotetext{
${ }^{6}$ The associative algebra (2.7) is the same as the semi-group $S_{E}^{(1)}=\left\{\lambda_{0}, \lambda_{1}, \lambda_{2}\right\}$ with $\lambda_{i} \cdot \lambda_{j}=\lambda_{j} \cdot \lambda_{i}=\lambda_{i+j}$ where $\lambda_{j}=0$ for $j>1$. One can use the Lie algebra expansion method [38-41] with this semi-group to obtain a new Lie algebra that is isomorphic to the Lie algebra we describe below. If we assign the hermiticity properties $\lambda_{i}^{\dagger}=\lambda_{i}$, then the Lie algebra obtained by the expansion method is exactly the same as we present in (2.13).
} 
The coloured Poincaré algebra then has the following commutation relations.

$$
\begin{aligned}
& {\left[M_{a}^{\widehat{I}}, M_{b}^{\widehat{J}}\right]=\frac{1}{2} \varepsilon_{a b}{ }^{c} \widehat{d}^{\widehat{I} \widehat{J}} \widehat{K} M_{c}^{\widehat{K}}-\frac{1}{4} \eta_{a b} \widehat{f} \widehat{I} \widehat{J} \widehat{K} N^{\widehat{K}},} \\
& {\left[M_{a}^{\widehat{I}}, N^{\widehat{J}}\right]=\widehat{f}^{\widehat{I J}}{ }_{\widehat{K}}^{\widehat{K}} M_{a}^{\widehat{K}},} \\
& {\left[N^{\widehat{I}}, N^{\widehat{J}}\right]=\widehat{f}^{\widehat{I J}} \widehat{K} N^{\widehat{K}},} \\
& {\left[M_{a}^{\widehat{I}}, P_{b}^{\widehat{J}}\right]=\frac{1}{2} \varepsilon_{a b}{ }^{c} \widehat{d}^{\widehat{I J}} \widehat{K} P_{c}^{\widehat{K}}-\frac{1}{4} \eta_{a b} \widehat{f}^{\widehat{I J}} \widehat{K} Q^{\widehat{K}},} \\
& {\left[M_{a}^{\widehat{I}}, Q^{\widehat{J}}\right]=\widehat{f}^{\widehat{I J}} \widehat{K} P_{a}^{\widehat{K}},} \\
& {\left[N^{\widehat{I}}, P_{a}^{\widehat{J}}\right]=\widehat{f}^{\widehat{I} \widehat{J}} \widehat{K} P_{a}^{\widehat{K}},} \\
& {\left[N^{\widehat{I}}, Q^{\widehat{J}}\right]=\widehat{f}^{\widehat{I} \widehat{J}} Q^{\widehat{K}},} \\
& {\left[P_{a}^{\widehat{I}}, P_{b}^{\widehat{J}}\right]=0,} \\
& {\left[P_{a}^{\widehat{I}}, Q^{\widehat{J}}\right]=0,} \\
& {\left[Q^{\widehat{I}}, Q^{\widehat{J}}\right]=0 \text {. }}
\end{aligned}
$$

The first three lines are the algebra $\mathfrak{u}(N, N)$ and the remaining lines can also be understood as the Lie algebra with the semi-group $S_{E}^{(1)}$ applied to $\mathfrak{u}(N, N)$, see footnote 6 .

We see from the commutation relations (2.13) that the $\widehat{I}=0$ components $M_{a}^{0}$ and $P_{a}^{0}$ satisfy the usual uncoloured Poincaré algebra and can be identified with these according to

$$
P_{a} \equiv P_{a}^{0}, \quad M_{a} \equiv M_{a}^{0} .
$$

The Lie algebra $\operatorname{cPoin}_{3}(N)$ has a two-dimensional center that can be quotiented out. This center is spanned by $N^{0}$ and $Q^{0}$. From the definition (2.11) we see that ${ }^{7}$

$$
\mathrm{cPoin}_{3} \cong \mathfrak{u}(N, N) \oplus_{\text {adj }} \mathfrak{u}(N, N),
$$

where the notation indicates a semi-direct sum of the Lie algebra $\mathfrak{u}(N, N)$ acting on another copy of the space $\mathfrak{u}(N, N)$, now treated as an abelian algebra, via the adjoint action. ${ }^{8}$ The Lie algebra $\mathfrak{u}(N, N)$ arises since $\mathfrak{u}(1,1) \otimes \mathfrak{u}(N) \cong \mathfrak{u}(N, N)$ upon using $\mathfrak{s l}(2, \mathbb{R}) \cong \mathfrak{s u}(1,1)$. The non-abelian first $\mathfrak{u}(N, N)$ in (2.15) is due to the idempotent $\mathcal{I}$ in (2.9) whereas the second abelian $\mathfrak{u}(N, N)$ is due to the nilpotent $\mathcal{J}$. The two-dimensional center in this language corresponds to the two $\mathfrak{u}(1)$ inside each of the two $\mathfrak{u}(N, N),{ }^{9}$ and we are left with

\footnotetext{
${ }^{7}$ We note that the result of the construction is the same as constructing the co-adjoint extension of $\mathfrak{u}(N, N)$ using [42, 43].

${ }^{8}$ In this notation, the usual uncoloured Poincaré algebra is written as the semi-direct sum $\mathfrak{s o}(1, D-1) €_{\text {vec }}$ $\mathbb{R}^{1, D-1}$ in any space-time dimension $D$. The space $\mathbb{R}^{1, D-1}$ is the abelian algebra of translations and acted upon by the Lorentz algebra using the $\mathfrak{s o}(1, D-1)$ vector representation.

${ }^{9}$ The presence of two $\mathfrak{u}(1)$ makes it possible to contract $\operatorname{cPoin}_{3}(N)$ to a non-relativistic colour algebra with a non-degenerate bilinear form, analogously to the uncolored case of the extended Bargmann algebra as a contraction of $\mathfrak{u}(1,1) \bigoplus_{\text {adj }} \mathfrak{u}(1,1)[21]$.
} 
the semi-direct sum

$$
\mathfrak{s u}(N, N) \oplus_{\text {adj }} \mathfrak{s u}(N, N)
$$

Because of the structure of (2.15) we shall refer to the generators $M_{a}^{\widehat{I}}$ and $N^{\widehat{I}}$ as generalised (coloured) Lorentz generators and to $P_{a}^{\widehat{I}}$ and $Q^{\widehat{I}}$ as generalised (coloured) translation generators that form an abelian subalgebra and whose eigenvalues are generalised momenta. As is evident from (2.15) and (2.16), the coloured Poincaré algebra is not a direct sum of the usual Poincaré algebra iso $(2,1)$ with an internal symmetry algebra. This is not in contradiction with the Coleman-Mandula theorem as we shall also enlarge the space-time beyond three dimensions when we construct particle actions below.

Since, according to (2.16), all objects are elements of $\mathfrak{s u}(N, N)$, it will be convenient to use an explicit matrix representation. In the defining representation of $\mathfrak{s u}(N, N)$ an element of the Lie algebra $\mathfrak{s u}(N, N)$ is written in block matrix form as

$$
\left(\begin{array}{ll}
A & B \\
C & D
\end{array}\right)
$$

with $N \times N$ blocks satisfying

$$
\mathbf{A}^{\dagger}=-\mathbf{A}, \quad \mathbf{B}^{\dagger}=\mathbf{C}, \quad \mathbf{C}^{\dagger}=\mathbf{B}, \quad \mathbf{D}^{\dagger}=-\mathbf{D}, \quad \operatorname{tr}(\mathbf{A}+\mathbf{D})=0 .
$$

We shall use bold letters to denote $(N \times N)$ matrices and 'tr' for the corresponding trace over $(N \times N)$-matrices. The identity $(N \times N)$ matrix is written as $\mathbf{I}$.

Coloured $\mathbf{A d S}_{\mathbf{2}}$ algebra. The first three lines of (2.13) are the $\mathfrak{u}(N, N)$ subalgebra of $\mathrm{cPoin}_{3}(N)$, explicitly

$$
\begin{aligned}
& {\left[M_{a}^{\widehat{I}}, M_{b}^{\widehat{J}}\right]=\frac{1}{2} \varepsilon_{a b}{ }^{c} \widehat{d}^{\widehat{I J}} \widehat{K} M_{c}^{\widehat{K}}-\frac{1}{4} \eta_{a b} \widehat{f} \widehat{I J} \widehat{K} N^{\widehat{K}},} \\
& {\left[M_{a}^{\widehat{I}}, N^{\widehat{J}}\right]=\widehat{f}_{\widehat{I} \widehat{K}}^{\widehat{I}} M_{a}^{\widehat{K}},} \\
& {\left[N^{\widehat{I}}, N^{\widehat{J}}\right]=\widehat{f}^{\widehat{I} \widehat{K}}{ }_{\widehat{K}}^{\widehat{K}} .}
\end{aligned}
$$

It can be understood as the tensor product algebra $\mathfrak{u}(1,1) \otimes \mathfrak{u}(N)$ :

$$
\begin{gathered}
M_{a}^{\widehat{I}}=-\mathrm{i} L_{a} \otimes T^{\widehat{I}}, \\
N^{\widehat{I}}=\mathbf{I}_{2} \otimes T^{\widehat{I}} .
\end{gathered}
$$

With a proper identification of the generators, this algebra is the coloured conformal algebra in one dimension or the coloured $\mathrm{AdS}_{2}$ algebra.

The basis of the coloured conformal algebra is given by

$$
D^{\widehat{I}}=M_{0}^{\widehat{I}}, \quad H^{\widehat{I}}=M_{1}^{\widehat{I}}+M_{2}^{\widehat{I}}, \quad K^{\widehat{I}}=M_{1}^{\widehat{I}}-M_{2}^{\widehat{I}} .
$$

The generators with the zero component in the colour indices give the uncoloured $D=1$ conformal algebra. 
The coloured $\mathrm{AdS}_{2}$ algebra can be written in terms of conformal generators

$$
P_{1}^{\widehat{I}}=\frac{1}{\ell} D^{\widehat{I}}, \quad P_{0}^{\widehat{I}}=\frac{1}{2 \ell}\left(H^{\widehat{I}}+K^{\widehat{I}}\right), \quad M_{0}^{\widehat{I}}=\frac{1}{2}\left(H^{\widehat{I}}-K^{\widehat{I}}\right) .
$$

The parameter $\ell$ denotes the radius of $\mathrm{AdS}_{2}$. The generators with the zero component in the colour indices give the uncoloured $\mathrm{AdS}_{2}$ algebra. This algebra can be used in a BF theory [44-47] to construct a coloured analogue of Jackiw-Teitelboim gravity (see the upcoming work [48] for a detailed analysis).

\section{Coloured gravity in $3 \mathrm{~d}$ Minkowski space-time}

The coloured Poincaré algebra introduced in the previous section can be understood as a zero cosmological constant limit of the coloured (A)dS 3 algebra [13], see also appendix A. Furthermore, one can define a Chern-Simons action for coloured gravity in 3d Minkowski space.

\subsection{Invariant bilinear form}

Let us recall the structure of the Poincaré algebra in three dimensions. As usual for Poincaré algebra in any dimensions, one can define a degenerate bilinear form

$$
\left\langle P_{a} \mid P_{b}\right\rangle=0, \quad\left\langle P_{a} \mid M_{b}\right\rangle=0, \quad\left\langle M_{a} \mid M_{b}\right\rangle=\eta_{a b} .
$$

In three dimensions, there is a different, non-degenerate bilinear form (see, e.g., [3]) which is, however, non-diagonal

$$
\left\langle P_{a} \mid P_{b}\right\rangle=0, \quad\left\langle P_{a} \mid M_{b}\right\rangle=\eta_{a b}, \quad\left\langle M_{a} \mid M_{b}\right\rangle=0 .
$$

The latter bilinear form allows us to define the Chern-Simons action for Poincaré algebra, equivalent to the Einstein-Hilbert action in three dimensions for an invertible dreibein. This is possible in three dimensions due to the fact that the Lorentz and translation generators of the Poincaré algebra carry the same Lorentz representation - they are both vectors of $\mathfrak{s o}(1,2)$, or, equivalently, are in a representation isomorphic to the adjoint representation of $\mathfrak{s u}(1,1) \simeq \mathfrak{s o}(1,2)$. This property generalises to the coloured Poincaré algebra, which has an invariant bilinear form with similar properties as we show below.

First we note that the most general bilinear form for the three-dimensional Poincaré algebra has a one-parameter freedom up to normalisation (see, e.g., [12]). Therefore, the most general Chern-Simons action based on three-dimensional Poincaré algebra can be given in the form,

$$
S=\frac{\kappa_{1}}{4 \pi} \int \operatorname{Tr}(e \wedge(d \omega+\omega \wedge \omega))+\frac{\kappa_{2}}{4 \pi} \int \operatorname{Tr}\left(\omega \wedge d \omega+\frac{2}{3} \omega \wedge \omega \wedge \omega\right),
$$

where we do not write the contractions of fiber Lorentz indices for simplicity. The second term in this action is the Lorentz-Chern-Simons term, which, supplemented with the torsion constraint describes conformal gravity in three dimensions. Vanishing of the torsion is a consequence of the equations of motion of the action (3.3). However, substituting the 
solution of the torsion constraint back into the action will change its physical content since the torsionless constraint is not a consequence of the $\omega$ equations alone for $\kappa_{2} \neq 0$. This situation is analogous to theories with "third-way consistency" [49]. If one nevertheless substitutes the solution of the torsion constraint back in the action, one gets so-called Topologically Massive Gravity (TMG) [50] with mass value $m \sim \kappa_{1} / \kappa_{2}$.

In order to construct an invariant bilinear form for the coloured Poincaré algebra, we first start from the coloured Lorentz subalgebra $\mathfrak{u}(N, N) \simeq \mathfrak{u}(1,1) \otimes \mathfrak{u}(N)$. For the colour group, $\mathfrak{u}(N)$, the bilinear form has the following form

$$
\left\langle N^{I} \mid N^{J}\right\rangle=\alpha \delta^{I J}, \quad\left\langle N^{I} \mid N^{0}\right\rangle=0, \quad\left\langle N^{0} \mid N^{0}\right\rangle=\beta,
$$

which is non-degenerate when $\alpha$ and $\beta$ are arbitrary non-zero numbers. A general ansatz for the rest of the generators, $M_{a}^{\hat{I}}=\left(M_{a}^{I}, M_{a}\right)$, can be given in the form:

$$
\left\langle M_{a}^{I} \mid M_{b}^{J}\right\rangle=\gamma \eta_{a b} \delta^{I J}, \quad\left\langle M_{a} \mid M_{b}\right\rangle=\delta \eta_{a b}
$$

The constraint of invariance of the bilinear form implies

$$
\gamma=\frac{1}{4} \alpha, \quad \delta=\frac{N}{4} \alpha
$$

As expected, the invariant bilinear form of the algebra $\mathfrak{u}(N, N)$ depends on two arbitrary parameters $\alpha, \beta$. One of these numbers parametrises the norm of the central element, which we can drop. Then, the bilinear form of the reduced coloured Lorentz algebra $\mathfrak{s u}(N, N)$ is fixed uniquely up to normalisation:

$$
\left\langle\mathcal{M}^{A} \mid \mathcal{M}^{B}\right\rangle=\rho \delta^{A B}, \quad \mathcal{M}^{A} \in \mathfrak{s u}(N, N),
$$

where $A, B=1,2, \ldots, 4 N^{2}-1$ denote here adjoint indices of $\mathfrak{s u}(N, N)$. The most general invariant bilinear form for the algebra $\mathfrak{s u}(N, N) \oplus_{\text {adj }} \mathfrak{s u}(N, N)$ is given in the following form.

$$
\left\langle\mathcal{M}^{A} \mid \mathcal{M}^{B}\right\rangle=\rho \delta^{A B}, \quad\left\langle\mathcal{M}^{A} \mid \mathcal{P}^{B}\right\rangle=\lambda \delta^{A B}, \quad\left\langle\mathcal{P}^{A} \mid \mathcal{P}^{B}\right\rangle=0,
$$

where $\rho$ and $\lambda$ are arbitrary numbers. This bilinear form is non-degenerate for $\lambda \neq 0$.

In analogy with (3.3), one can write a Chern-Simons action for coloured Poincaré gravity in three dimensions in the following schematic form.

$$
S=\frac{\kappa}{4 \pi} \int \operatorname{Tr}(E \wedge(d \Omega+\Omega \wedge \Omega))+\frac{\tilde{\kappa}}{4 \pi} \int \operatorname{Tr}\left(\Omega \wedge d \Omega+\frac{2}{3} \Omega \wedge \Omega \wedge \Omega\right)
$$

where the fields $E, \Omega$ constitute the coloured Poincaré connection,

$$
\mathcal{A}=E^{A} \mathcal{P}_{A}+\Omega^{A} \mathcal{M}_{A} .
$$

Naively, solving the torsion constraint,

$$
T^{A}=d E^{A}+\frac{1}{2} f^{A}{ }_{B C}\left(\Omega^{B} \wedge E^{C}+E^{B} \wedge \Omega^{C}\right)=0,
$$


for $\Omega=\Omega(E)$ and plugging back into the action (3.9), one will get the coloured analogue of TMG. This substitution, even if possible technically, is leading to a different physical theory which we will not study here.

The space-time parity transformation multiplies odd space-time forms by -1 and transforms the coloured Poincaré algebra generators as $\left(\mathcal{P}_{A}, \mathcal{M}_{A}\right) \rightarrow\left(-\mathcal{P}_{A}, \mathcal{M}_{A}\right)$. Consequently it acts on the one-form fields as $(E, \Omega) \rightarrow(E,-\Omega)$ while $(d E, d \Omega) \rightarrow(-d E, d \Omega)$. Under this action the first term of (3.9) is invariant, while the second term changes sign.

For constructing the theory that is even under space-time parity, one makes use of the non-diagonal bilinear form ( $\rho=0$ case), which can be given in the following form (we write only non-zero scalar products).

$$
\left\langle M_{a}^{I} \mid P_{b}^{J}\right\rangle=\frac{1}{4} \lambda \eta_{a b} \delta^{I J}, \quad\left\langle N^{I} \mid Q^{J}\right\rangle=\lambda \delta^{I J}, \quad\left\langle M_{a} \mid P_{b}\right\rangle=\frac{N}{4} \lambda \eta_{a b} .
$$

Using this bilinear form, one can write a Chern-Simons action with coloured Poincaré symmetry, that will be equivalent to the flat limit of coloured gravity in (A) $\mathrm{dS}_{3}$, studied in [13]. It is natural to choose $\lambda=\frac{4}{N}$ to recover $\left\langle M_{a} \mid P_{b}\right\rangle=\eta_{a b}$ from [3] (the overall constant is incorporated in the Chern-Simons level).

\subsection{Coloured gravity action in 3d Minkowski space}

As discussed above, the action for coloured gravity in three-dimensional Minkowski spacetime can be given in the Chern-Simons form with coloured Poincaré gauge symmetry, using the non-degenerate bilinear form. There is one choice of the bilinear form, (3.12), that reproduces (3.2) for the uncoloured Poincaré subalgebra. We will use (3.12) to reproduce Einstein-Hilbert gravity for the $\mathfrak{s u}(N)$ singlet spin-two field.

Coloured Poincaré gravity can be defined by a Chern-Simons action,

$$
S[\mathcal{A}]=\frac{\kappa}{4 \pi} \int \operatorname{Tr}\left(\mathcal{A} \wedge d \mathcal{A}+\frac{2}{3} \mathcal{A} \wedge \mathcal{A} \wedge \mathcal{A}\right),
$$

where $\mathcal{A} \in \mathfrak{s u}(N, N) \oplus_{\text {adj }} \mathfrak{s u}(N, N)$ (we factor by the center since it decouples in the action). In analogy with [13], we will further decompose $\mathcal{A}$ as

$$
\mathcal{A}=\mathcal{B}+\mathcal{C}
$$

where $\mathcal{B}$ incorporates the uncoloured isometry and colour group: ${ }^{10}$

$$
\mathcal{B}=\omega^{a} M_{a}+e^{a} P_{a}+\varphi_{I} N^{I}+\tilde{\varphi}_{I} Q^{I},
$$

while $\mathcal{C}$ corresponds to the coloured spin-two sector:

$$
\mathcal{C}=\omega_{I}^{a} M_{a}^{I}+e_{I}^{a} P_{a}^{I} .
$$

The action then can be written in the form,

$$
S[\mathcal{B}, \mathcal{C}]=\frac{\kappa}{4 \pi} \int \operatorname{Tr}\left(\mathcal{B} \wedge d \mathcal{B}+\frac{2}{3} \mathcal{B} \wedge \mathcal{B} \wedge \mathcal{B}+\mathcal{C} \wedge D_{\mathcal{B}} \mathcal{C}+\frac{2}{3} \mathcal{C} \wedge \mathcal{C} \wedge \mathcal{C}\right)
$$

\footnotetext{
${ }^{10}$ Here the one-form field $\omega$ is related to the standard spin-connection as $\omega^{a}=\frac{1}{2} \epsilon^{a b c} \omega_{b c}$. Similarly for its $\mathfrak{s u}(N)$-valued analogue, $\omega_{I}^{a}$.
} 
with

$$
D_{\mathcal{B}} \mathcal{C}=d \mathcal{C}+\mathcal{B} \wedge \mathcal{C}+\mathcal{C} \wedge \mathcal{B}
$$

The sector that depends only on $\mathcal{B}$ can be explicitly written in the form,

$$
S_{\mathcal{B}}=\frac{\kappa}{4 \pi} \int \operatorname{Tr}\left(\mathcal{B} \wedge d \mathcal{B}+\frac{2}{3} \mathcal{B} \wedge \mathcal{B} \wedge \mathcal{B}\right)=S_{\mathrm{GR}}+S_{\text {Vector }}
$$

with

$$
\begin{aligned}
S_{\mathrm{GR}} & =\frac{\kappa}{2 \pi} \int e^{a} \wedge\left(d \omega^{b}+\frac{1}{2} \epsilon^{b c d} \omega_{c} \wedge \omega_{d}\right) \eta_{a b}, \\
S_{\text {Vector }} & =\frac{2 \kappa}{N \pi} \int \tilde{\varphi}^{I} \wedge\left(d \varphi^{J}+f^{J}{ }_{K L} \varphi^{K} \wedge \varphi^{L}\right) \delta_{I J} .
\end{aligned}
$$

For zero cosmological constant, as opposed to the $(\mathrm{A}) \mathrm{dS}_{3}$ case, the rewriting of the action in terms of chiral and anti-chiral pieces is not possible neither in the pure gravity sector, $S_{\mathrm{GR}}$, nor the vector field sector, $S_{\text {Vector }}$. The equations of motion for the vector sector (neglecting contribution from interactions with coloured gravitons) can be given as

$$
\begin{aligned}
F(\varphi)=d \varphi+\varphi \wedge \varphi & =0, \\
D_{\varphi} \tilde{\varphi}=d \tilde{\varphi}+\varphi \wedge \tilde{\varphi}+\tilde{\varphi} \wedge \varphi & =0,
\end{aligned}
$$

and corresponds to a BF theory (see, e.g., [51-53]). For non-zero cosmological constant, $\Lambda \neq 0$, the Lagrangian of the GR sector would get an additional piece $\sim \Lambda e \wedge e \wedge e$, and the gauge vector field sector would get an additional piece $\sim \Lambda \tilde{\varphi} \wedge \tilde{\varphi} \wedge \tilde{\varphi}$, both essential for diagonalisation of the theory and deformation of the algebra.

The action of the matter sector has the following form,

$$
S_{\mathcal{C}}=\frac{\kappa}{4 \pi} \int \operatorname{Tr}\left(\mathcal{C} \wedge D_{\mathcal{B}} \mathcal{C}+\frac{2}{3} \mathcal{C} \wedge \mathcal{C} \wedge \mathcal{C}\right)
$$

or, in a more explicit form,

$$
\begin{aligned}
S_{\mathcal{C}}=\frac{\kappa}{2 N \pi} \int & \left(\delta^{I J} \eta_{a b} e_{I}^{a} \wedge d \omega_{J}^{b}+\frac{1}{2} \delta^{I J} \epsilon_{a b c} \omega_{I}^{a} \wedge \omega_{J}^{b} \wedge e^{c}\right. \\
& +\delta^{I J} \epsilon_{a b c} \omega_{I}^{a} \wedge e_{J}^{b} \wedge \omega^{c}+\frac{1}{4} d^{I J K} \epsilon_{a b c} \omega_{I}^{a} \wedge \omega_{J}^{b} \wedge e_{K}^{c} \\
& \left.-f^{I J K} \eta_{a b} \omega_{I}^{a} \wedge e_{J}^{b} \wedge \varphi_{K}-\frac{1}{2} f^{I J K} \eta_{a b} \omega_{I}^{a} \wedge \omega_{J}^{b} \wedge \tilde{\varphi}_{K}\right) .
\end{aligned}
$$

It is interesting to note that the multiple background solutions with different cosmological constants of coloured gravity in $(\mathrm{A}) \mathrm{dS}_{3}$ [13] all go to the Minkowski solution in the $\Lambda \rightarrow 0$ limit. 
The second term of (3.9), the coloured Lorentz-Chern-Simons term, is also straightforward to compute:

$$
\begin{aligned}
S_{\mathrm{CLCS}}= & \frac{\tilde{\kappa}}{4 \pi} \int\left(\omega^{a} \wedge d \omega^{b} \eta_{a b}+\frac{1}{3} \epsilon_{a b c} \omega^{a} \wedge \omega^{b} \wedge \omega^{c}\right) \\
+ & +\frac{\tilde{\kappa}}{N \pi} \int\left(\varphi^{I} \wedge d \varphi^{J} \delta_{I J}+\frac{2}{3} f_{I J K} \varphi^{I} \wedge \varphi^{J} \wedge \varphi^{K}\right) \\
+ & +\frac{\tilde{\kappa}}{4 N \pi} \int\left(\delta^{I J} \eta_{a b} \omega_{I}^{a} \wedge d \omega_{J}^{b}+\frac{1}{6} d^{I J K} \epsilon_{a b c} \omega_{I}^{a} \wedge \omega_{J}^{b} \wedge \omega_{K}^{c}\right. \\
& \left.+\delta^{I J} \epsilon_{a b c} \omega_{I}^{a} \wedge \omega_{J}^{b} \wedge \omega^{c}-f^{I J K} \eta_{a b} \omega_{I}^{a} \wedge \omega_{J}^{b} \wedge \varphi_{K}\right) .
\end{aligned}
$$

\section{Free coloured particle model}

In this section, we are considering massive and massless particle actions invariant under the coloured Poincaré algebra. First we introduce the coloured generalisation of Minkowski space and then discuss how the different ways of constructing Poincaré particle actions become coloured. In our analysis we shall consistently quotient by the two-dimensional center generated by $N^{0}$ and $Q^{0}$ and thus restrict to $\mathfrak{s u}(N, N) €_{\text {adj }} \mathfrak{s u}(N, N)$ as given in $(2.16)$.

\subsection{Coloured Minkowski space}

The algebra $\mathrm{cPoin}_{3}(N)$ is a vast extension of the Poincaré algebra (2.5). Its centerless version (2.16) includes an abelian part spanned by $P_{a}, P_{a}^{I}$ and $Q^{I}$. This should be thought of as coloured translations and there is an associated coloured Minkowski space. Introducing dual coordinates for each of the coloured translation generators we obtain coordinates $x^{a}$, $x_{I}^{a}$ and $y_{I}$, where the first $x^{a}$ is to be thought of as the usual Minkowski coordinate (invariant under $\mathfrak{s u}(N)$ and the others are the coloured extensions. Similarly, the coloured Lorentz algebra $\mathfrak{s u}(N, N)$ has generators $M_{a}, M_{a}^{I}$ and $N^{I}$, where the first generator is to be thought of as the usual Lorentz generator and the others as coloured generalisations.

On a coloured Minkowski point $\left(x^{a}, x_{I}^{a}, y_{I}\right)$ represented by

$$
\text { Х }=x^{a} P_{a}+x_{I}^{a} P_{a}^{I}+y_{I} Q^{I},
$$

an infinitesimal coloured Poincaré transformation is obtained from the Lie algebra element $(\mathbb{O}, \mathbb{A}) \in \mathfrak{s u}(N, N) \oplus_{\text {adj }} \mathfrak{s u}(N, N)$ with $^{11}$

$$
\mathbb{O}=\underbrace{\omega^{a} M_{a}+\omega_{I}^{a} M_{a}^{I}+\sigma_{I} N^{I}}_{\text {coloured Lorentz }}, \quad \mathbb{A}=\underbrace{\alpha^{a} P_{a}+\alpha_{I}^{a} P_{a}^{I}+\beta_{I} Q^{I}}_{\text {coloured translations }}
$$

and produces the linear transformation,

$$
\delta \mathbb{X}=[\mathbb{O}, \mathbb{X}]+\mathbb{A} .
$$

\footnotetext{
${ }^{11}$ The notation $\omega$ for the parameter here should not be confused with the gauge fields $\omega$ from section 3 .
} 
More explicitly, the transformation rule reads

$$
\begin{aligned}
\delta x^{a} & =\varepsilon_{b c}{ }^{a} \omega^{b} x^{c}+\frac{1}{N} \delta^{I J} \varepsilon_{b c}{ }^{a} \omega_{I}^{b} x_{J}^{c}+\alpha^{a}, \\
\delta x_{I}^{a} & =\varepsilon_{b c}{ }^{a} \omega^{b} x_{I}^{c}+\varepsilon_{b c}{ }^{a} \omega_{I}^{b} x^{c}+\frac{1}{2} \varepsilon_{b c}{ }^{a} d^{J K}{ }_{I} \omega_{J}^{b} x_{K}^{c}+f^{J K}{ }_{I} \omega_{J}^{a} y_{K}+f^{J K}{ }_{I} \sigma_{J} x_{K}^{a}+\alpha_{I}^{a}, \\
\delta y_{I} & =-\frac{1}{4} \eta_{a b} f^{J K}{ }_{I} \omega_{J}^{a} x_{K}^{b}+f^{J K}{ }_{I} \sigma_{J} y_{K}+\beta_{I} .
\end{aligned}
$$

Here, we have used (2.13).

The usual Lorentz part $\omega^{a}$ acts on the usual coordinate $x^{a}$ in the standard way. The coloured Lorentz generators $N^{I}$, that satisfy the $\mathfrak{s u}(N)$ algebra according to $(2.13 \mathrm{c})$, generate colour rotations on the colour-extended coordinates $x_{I}^{a}$ and $y_{I}$. Note that these coordinates are in the adjoint of $\mathfrak{s u}(N) .{ }^{12}$

The generators of coloured Lorentz group are obtained by tensoring such matrices with $\mathcal{I}$ and the generators of the coloured translations by tensoring with $\mathcal{J}$, see $(2.7)$ and (2.12). We shall suppress the tensoring with these elements consistently. The action of the generalised Lorentz group on generalised Minkowski space can just be written as matrix operations.

In the representation (2.17), a point on coloured Minkowski space is written as

$$
\mathbf{X}=\left(\begin{array}{cc}
\mathrm{i} \mathbf{X}^{0}+\mathrm{i} \mathbf{Y} & \mathbf{X}^{+} \\
\mathbf{X}^{-} & -\mathrm{i} \mathbf{X}^{0}+\mathrm{i} \mathbf{Y}
\end{array}\right)
$$

with $\mathbf{X}^{ \pm}=\mathbf{X}^{1} \pm \mathrm{i} \mathbf{X}^{2}$ and

$$
\mathbf{X}^{a}=x^{a} \mathbf{I}+\mathrm{i} x_{I}^{a} T^{I}, \quad \mathbf{Y}=\mathrm{i} y_{I} T^{I} .
$$

All coefficients are real-valued, thus $\left(\mathbf{X}^{ \pm}\right)^{\dagger}=\mathbf{X}^{\mp}$. Writing the parameter of a transformation $(4.2)$ as

$$
\mathbb{O}=\left(\begin{array}{cc}
\mathrm{i} \omega^{0}+\mathrm{i} \boldsymbol{\sigma} & \boldsymbol{\omega}^{+} \\
\omega^{-} & -\mathrm{i} \omega^{0}+\mathrm{i} \boldsymbol{\sigma}
\end{array}\right), \quad \mathbb{A}=\left(\begin{array}{cc}
\mathrm{i} \boldsymbol{\alpha}^{0}+\mathrm{i} \boldsymbol{\beta} & \boldsymbol{\alpha}^{+} \\
\boldsymbol{\alpha}^{-} & -\mathrm{i} \boldsymbol{\alpha}^{0}+\mathrm{i} \boldsymbol{\beta}
\end{array}\right),
$$

with component expansions similar to above, the transformation in (4.3) corresponds exactly to the matrix commutator.

A metric on coloured Minkowski space that is invariant under coloured Lorentz transformations is given by

$$
\frac{1}{2 N} \operatorname{Tr}\left(\bigvee^{2}\right)=x^{a} x^{b} \eta_{a b}+\frac{1}{N} x_{I}^{a} x_{J}^{b} \eta_{a b} \delta^{I J}-\frac{1}{N} y_{I} y_{J} \delta^{I J},
$$

where $\eta_{a b}$ is the $(-++)$ Minkowski metric and $\delta^{I J}$ the $\mathrm{SU}(N)$ invariant metric. We see that it consists of the usual Minkowski metric for the coordinates $x^{a}$ and the other coordinates are suppressed by factors of $1 / N$. Using the invariant metric (4.8) one can define the notion

\footnotetext{
${ }^{12}$ It is amusing to observe that the transformation of the usual Minkowski coordinates $x^{a}$ does not depend on the remaining coordinates in the limit $N \rightarrow \infty$.
} 
of coloured null, time-like and space-like vectors in the usual way depending on whether the norm is zero, negative or positive, respectively.

For writing particle actions, we shall also make use of the matrix notation (4.5) and moreover require a matrix representation of the (conjugate) momentum that we write as

$$
\mathbb{P}=\left(\begin{array}{cc}
\mathrm{i} \mathbf{P}^{0}+\mathrm{i} \boldsymbol{\Pi} & \mathbf{P}^{+} \\
\mathbf{P}^{-} & -\mathrm{i} \mathbf{P}^{0}+\mathrm{i} \boldsymbol{\Pi}
\end{array}\right) .
$$

The momentum vector $\mathbb{P}$ transforms in the coadjoint of the coloured Lorentz algebra $\mathfrak{s u}(N, N)$, thus $\mathbb{P} \in \mathfrak{s u}(N, N)^{*}: \delta_{\mathbb{O}} \mathbb{P}=[\mathbb{O}, \mathbb{P}]$. The components satisfy $\left(\mathbf{P}^{ \pm}\right)^{\dagger}=\mathbf{P}^{\mp}$ and $\boldsymbol{\Pi}$ is traceless and hermitian. Expanding out these matrices explicitly we write

$$
\mathbf{P}^{a}=p^{a} \mathbf{I}+\mathrm{i} p_{I}^{a} T^{I}, \quad \mathbf{\Pi}=\mathrm{i} \pi_{I} T^{I} .
$$

We note that in the uncoloured case $N=1$ we have

$$
N=1: \quad \mathbb{P}^{2}=\left(\begin{array}{cc}
p^{a} p_{a} & 0 \\
0 & p^{a} p_{a}
\end{array}\right)=p^{a} p_{a} \rrbracket .
$$

That $\mathbb{P}^{2}$ is proportional to the identity matrix is unique to the case $N=1$.

We shall moreover need a matrix Lagrange multiplier $\mathbb{L}$ whose matrix form we take as

$$
\mathbb{L}=-\mathrm{i}\left(\begin{array}{cc}
\mathrm{i} \mathbf{K}^{0}+\mathrm{i} \boldsymbol{\Lambda} & \mathbf{K}^{+} \\
\mathbf{K}^{-} & -\mathrm{i} \mathbf{K}^{0}+\mathrm{i} \boldsymbol{\Lambda}
\end{array}\right)
$$

that satisfies $\left(\mathbf{K}^{ \pm}\right)^{\dagger}=\mathbf{K}^{\mp}$ and where $\boldsymbol{\Lambda}$ is now traceful and hermitian. In other words $\mathrm{i} \mathbb{L} \in \mathfrak{u}(N, N)$ and we have multiplied by a convenient $-\mathrm{i}$ for simplifying certain expressions later. Expanding out the matrices we write

$$
\mathbf{K}^{a}=f^{a} \mathbf{I}+\mathrm{i} k_{I}^{a} T^{I}, \quad \mathbf{\Lambda}=e \mathbf{I}+\mathrm{i} \lambda_{I} T^{I} .
$$

The extra $\mathfrak{u}(1)$ component corresponds to the parameter $e$.

\subsection{Different types of massive particle actions}

Before discussing particle actions for coloured Minkowski space we briefly review different particle actions in uncoloured Minkoswki space and how they are related.

\subsubsection{Uncoloured Minkowski space}

In ordinary Minkowski space with coordinates $x^{a}$, there are several ways of writing the action for a massive particle of mass $m>0$.

1. Geometric action.

$$
S_{\text {geo }}^{\text {unc. }}\left[x^{a}\right]=-m \int d \tau \sqrt{-\dot{x}^{a} \dot{x}^{b} \eta_{a b}} \text {. }
$$


This action describes the proper length of the time-like world-line $\left(\dot{x}^{a} \dot{x}^{b} \eta_{a b}<0\right)$, where the dot denotes differentiation with respect to the arbitrary world-line parameter $\tau$. The action is invariant under general world-line reparametrisations. We note that the momentum conjugate to $x^{a}$ that follows from this action is

$$
p_{a}=\frac{m \dot{x}_{a}}{\sqrt{-\dot{x}^{b} \dot{x}^{c} \eta_{b c}}} \quad \Longrightarrow \quad p_{a} p^{a}+m^{2}=0 .
$$

The last equation is the usual mass-shell constraint for a massive particle. In proper time gauge $\dot{x}^{a} \dot{x}_{a}=-1$, the equations of motion are simply $\ddot{x}^{a}=0$.

2. Hamiltonian action.

$$
S_{\mathrm{Ham}}^{\text {unc. }}\left[x^{a}, p_{a}, e\right]=\int d \tau\left(\dot{x}^{a} p_{a}+e\left(p^{a} p_{a}+m^{2}\right)\right) .
$$

This action utilises the einbein $e$ and the (conjugate) momentum $p_{a}$, both of which appear algebraically and are fully independent variables. By first integrating out $p^{a}$ and then $e$ by using their equations of motion one re-obtains (4.14). Since the equation for $e$ is quadratic there are two solutions, corresponding to changing the overall sign of (4.14). The mass-shell constraint,

$$
p^{a} p_{a}+m^{2}=0
$$

obtained by varying (4.16) with respect to $e$ has two independent solutions (up to the action of the orthochronous Lorentz group) corresponding to positive and negative energy particles that are distinguished by the sign of $p^{0}$. More abstractly, there are two independent semi-simple orbits of the action of $\mathrm{SO}(2,1)$ on the space of momenta $p^{a}$ that satisfy the uncoloured mass-shell constraint (4.17).

If we write the uncoloured Minkowski coordinates in matrix form using (4.5), we can also write this action as

$$
S_{\text {Ham }}^{\text {unc. }}[\mathbb{X}, \mathbb{P}, e]=\frac{1}{2} \int d \tau \operatorname{Tr}\left[\mathbb{P} \dot{\mathcal{X}}+e\left(\mathbb{P}^{2}+m^{2} \llbracket\right)\right],
$$

where we have also used (4.9) and (4.11). Since for $N=1, \mathbb{P}^{2}=p^{a} p_{a} \rrbracket$ we can even write the very same action in the form

$$
S_{\text {Ham }}^{\text {unc. }}[\mathbb{X}, \mathbb{P}, \mathbb{L}]=\frac{1}{2} \int d \tau \operatorname{Tr}\left[\mathbb{P} \dot{\mathcal{X}}+\mathbb{L}\left(\mathbb{P}^{2}+m^{2} \llbracket\right)\right],
$$

where $\mathbb{L}$ is now a matrix of Lagrange multipliers as introduced in (4.12) with $\frac{1}{2} \operatorname{Tr} \mathbb{L}=e$.

3. Orbit action.

$$
S_{\text {orb }}^{\text {unc. }}\left[x^{a}, \vec{p}\right]=\int d \tau \dot{x}^{a} p_{a}, \quad p_{0}= \pm \sqrt{\vec{p}^{2}+m^{2}},
$$

where the condition $p_{0}= \pm \sqrt{\vec{p}^{2}+m^{2}}$ selects only one massive orbit, depending on the sign one chooses. This action is in a sense the simplest action one can write for any given co-adjoint orbit, here again restricted to semi-simple orbits since $p^{a} p_{a}+m^{2}=0$ by construction. This action also follows from the method of non-linear realisation as we discuss in more detail in appendix B. 
The number of degrees of freedom can be determined from the actions in all three cases and one finds two degrees of freedom (in configuration space). This number of degrees of freedom agrees also with the dimension of the orbit of a massive momentum under $\mathrm{SO}(1,2)$. Even though there are two distinct orbits, corresponding to hyperboloids in the forward and backward light-cone or positive and negative energy particles, respectively, the dimension of all these orbits is the same. ${ }^{13}$

For the case of the massless particle one can most easily proceed from the Hamiltonian action (4.16) which is perfectly well-behaved in the limit $m \rightarrow 0$. The mass-shell constraint $p^{a} p_{a}=0$ now selects nilpotent orbits in momentum space, corresponding to the light-cones themselves. Imposing the corresponding orbit condition $p_{0}= \pm \sqrt{\vec{p}^{2}}$ in (4.20) describes the same physics.

Thinking of an (irreducible) particle as corresponding to a single momentum orbit, the most natural action is (4.20) as it only contains a single orbit whereas both (4.14) and (4.16) collect several orbits together. While for the uncoloured Poincaré algebra, there are only two distinct massive orbits (of positive and negative energy) and the distinction may seem innocuous, we shall see next that for the coloured Poincaré algebra the distinction is more important.

\subsubsection{Coloured Minkowski space}

1. Geometric action. We have determined the invariant line element in (4.8) and can therefore immediately write a world-line reparametrisation invariant action for a massive particle

$$
S_{\text {geo }}[\mathbb{X}]=-m \int d \tau \sqrt{-\frac{1}{2 N} \operatorname{Tr}\left[\dot{\boldsymbol{X}}^{2}\right]}
$$

The conjugate momentum is given by

$$
\mathbb{P}=\frac{1}{2 N} m \frac{\dot{\mathfrak{X}}^{T}}{\left.\sqrt{-\frac{1}{2 N} \operatorname{Tr}\left[\dot{\mathfrak{X}}^{2}\right.}\right]}
$$

that satisfies the constraint

$$
\operatorname{Tr}\left[\mathbb{P}^{2}+m^{2} \square\right]=0,
$$

where $\llbracket$ is the $2 N \times 2 N$ unit matrix. This scalar constraint is the generator of worldline diffeomporphisms. The time-like nature of the coloured velocity corresponds to $-\operatorname{Tr}\left[\dot{\mathbb{K}}^{2}\right]>0$. The equations of motion implied by this geometric action in proper time gauge are simply

$$
\ddot{\mathbf{X}}=0 \text {. }
$$

The proper time gauge condition implies $-\frac{1}{2 N} \operatorname{Tr}\left[\dot{\mathfrak{K}}^{2}\right]=1$.

\footnotetext{
${ }^{13}$ All these actions take exactly the same form in $D$-dimensional space-time, where the massive particle has $D-1$ degrees of freedom.
} 
2. Hamiltonian action. In order to write the Hamiltonian action we now have several expressions that could be generalised. The canonical action that corresponds directly to the geometric action above is

$$
\tilde{S}_{\text {Ham }}[\mathbb{X}, \mathbb{P}, e]=\frac{1}{2 N} \int d \tau \operatorname{Tr}\left[\mathbb{P} \dot{\mathcal{X}}+e\left(\mathbb{P}^{2}+m^{2} \square\right)\right],
$$

and it is the direct generalisation of (4.18). Integrating out $\mathbb{P}$ and $e$ from this action is not straight-forward and we discuss some aspects of this in appendix D. By varying (4.25) with respect to $e$, we re-obtain the scalar constraint (4.23). There are many solutions to this scalar constraint, corresponding to a continuum of different semi-simple orbits. These orbits can even have different dimensions and thus describe particles with different numbers of degrees of freedom. We discuss aspects of these orbits in more detail below in section 4.3 .

In order to always have massive particles with the same number of degrees of freedom, we can alternatively consider the generalisation of (4.19) that becomes

$$
S_{\text {Ham }}[\mathbb{X}, \mathbb{P}, \mathbb{L}]=\frac{1}{2 N} \int d \tau \operatorname{Tr}\left[\mathbb{P} \dot{\mathcal{X}}+\mathbb{L}\left(\mathbb{P}^{2}+m^{2} \llbracket\right)\right],
$$

now with a matrix-valued Lagrangian multiplier $\mathbb{L}$ as written out in (4.12). Note that i $\mathbb{L} \in \mathfrak{u}(N, N)$ and therefore there is a non-vanishing component $\frac{1}{2 N} \operatorname{Tr}[\mathbb{L}]=e$. The constraint that the matrix-valued Lagrange multipliers imposes is

$$
\mathbb{P}^{2}+m^{2} \square=0
$$

which is a $(2 N) \times(2 N)$ matrix condition. The scalar constraint (4.23) is its trace and therefore a weaker condition; only in the case $N=1$ the conditions are equivalent by virtue of (4.11).

The matrix constraint (4.27) restricts the possible momentum orbit more strongly than the trace constraint and, in particular, all semi-simple orbits satisfying (4.27) have the same dimension as we shall review in more detail in section 4.3.

3. Orbit action. In analogy with (4.20), we can also consider the simple action associated with a given orbit. This corresponds to considering

$$
S_{\text {orb }}=\frac{1}{2 N} \int d \tau \operatorname{Tr}[\mathbb{P} \dot{\mathbf{X}}]=\int d \tau\langle\mathbb{P}, \dot{\mathbf{X}}\rangle, \quad \mathbb{P} \text { belonging to a given massive orbit. }
$$

In the second step we have introduced the canonical pairing between $\mathfrak{s u}(N, N)$ and its dual: for any element $\mathcal{B} \in \mathfrak{s u}(N, N)^{*}$, there is a unique element $H_{\mathcal{B}} \in \mathfrak{s u}(N, N)$ satisfying

$$
\langle\mathcal{B}, A\rangle=\frac{1}{2 N} \operatorname{Tr}\left[A H_{\mathcal{B}}\right], \quad \text { for all } A \in \mathfrak{s u}(N, N),
$$

where the trace is for the fundamental representation of $\mathfrak{s u}(N, N)$. By abuse of notation, we write $\mathbb{P}$ both for the adjoint and the coadjoint representative. 
We briefly explain in this case why the orbit action agrees with the action one would derive from a standard non-linear realisation [54-57] for a semi-direct product. We do this for simplicity by focussing on the case when a representative of the massive orbit is given by

$$
\mathbb{P}=m \mathcal{P}_{0}^{0}=m\left(\begin{array}{cc}
\mathrm{i} \mathbf{I} & 0 \\
0 & -\mathrm{i} \mathbf{I}
\end{array}\right),
$$

where $\mathcal{P}_{0}^{0} \in \mathfrak{s u}(N, N)^{*}$ is the generator dual to $P_{0}^{0}$, the usual momentum. The stabiliser of this momentum is generated by

$$
M_{0} \equiv M_{0}^{0}, \quad M_{0}^{I}, \quad N^{I}
$$

as can be checked using the algebra (2.11). The algebra generated by (4.31) is $\mathfrak{u}(1) \oplus \mathfrak{s u}(N) \oplus \mathfrak{s u}(N)$. We thus take $H=(\mathrm{U}(1) \times \mathrm{SU}(N) \times \mathrm{SU}(N))$ and write a local representative of the coset in the form

$$
g=e^{\nwarrow} b h,
$$

with

$\mathcal{X}=x^{a} P_{a}+x_{I}^{a} P_{a}^{I}+y_{I} Q^{I} \quad$ (generalised coordinate of coloured Minkowski space),

$$
b=\exp \left[v^{i} M_{i}+w_{I}^{i} M_{i}^{I}\right] \quad \text { (generalised boost), }
$$

and $h$ belonging to the generalised little group $H$ generated by (4.31). The indices $a=0,1,2$ are covariant while $i=1,2$ are purely spatial indices. The generalised boost $b$ represents the broken generalised Lorentz generators acting on the generalised coordinate $\mathbf{X}$. We think of $x^{a}$ as the uncoloured position variable and $x_{I}^{a}$ and $y_{I}$ as coloured generalisation required by the algebra in the way described in section 4.1. All coordinates here are real.

The Maurer-Cartan form associated with the coset representative (4.32) is

$$
\Omega=g^{-1} d g=b^{-1} d \ b+b^{-1} d b
$$

where we have chosen the gauge $h=1$. The component of $\Omega$ along $P_{0}$ is invariant under the little group and its pullback to the world-line can be used as a Lagrangian that by construction then will be invariant under the full coloured Poincaré group. Using the pairing with the dual space of the coloured translation

$$
L_{\mathrm{nlr}} d t=m\left[\Omega^{*}\right]_{P_{0}}=\left\langle m \mathcal{P}_{0}, b^{-1} d \mathbb{X} b\right\rangle,
$$

where $\langle\cdot, \cdot\rangle$ is the pairing of the Lie algebra with its dual and $\mathcal{P}_{0}$ is the dual of the generator $P_{0}$. Now, using the invariance of the pairing, we can rewrite this as

$$
L_{\mathrm{nlr}}=\left\langle\operatorname{Ad}_{b}^{*}\left(m \mathcal{P}_{0}\right), \frac{d \mathbb{Z}}{d t}\right\rangle=\langle\mathbb{P}, \dot{\mathcal{X}}\rangle,
$$


where now $\mathbb{P}$ is any element of the orbit of the reference momentum. ${ }^{14}$ We thus have shown that the free particle Lagrangian obtained from the non-linear realisation is equal to that of the orbit construction (4.28), see also appendix B.

\subsection{Coadjoint orbits of $\mathfrak{s u}(N, N)$}

The coloured Lorentz group $\mathrm{SU}(N, N)$ acts on the momentum $\mathbb{P} \in \mathfrak{s u}(N, N)^{*}$ using the coadjoint action. In the above actions we have seen that the matrix constraint

$$
\mathbb{P}^{2}+m^{2} \square=0
$$

and the scalar constraint

$$
\operatorname{Tr}\left[\mathbb{P}^{2}+m^{2} \square\right]=0
$$

arose. These constraints are both $\mathrm{SU}(N, N)$ covariant and the solutions can therefore be classified in terms of coadjoint orbits. Since $\mathfrak{s u}(N, N)$ is simple we can identity coadjoint orbits with adjoint orbits using the non-degenerate trace pairing (4.29). For a general discussion of orbits see [58].

\subsubsection{Massive particles: semi-simple orbits}

For $m>0$, the relevant orbits are of semi-simple type and have representatives of the form

$$
\mathbb{P}=\operatorname{diag}\left(a_{1}, \ldots, a_{N}, b_{1} \ldots, b_{N}\right)
$$

The condition $\mathbb{P} \in \mathfrak{s u}(N, N)^{*}$ implies that $\sum_{j=1}^{n}\left(a_{j}+b_{j}\right)=0$ and our hermiticity conditions (2.18) imply that the $a_{j}$ and $b_{j}$ are pure imaginary. Interchanging $a_{j} \leftrightarrow a_{k}$ or $b_{j} \leftrightarrow b_{k}$ for the representative does not change the orbit.

Imposing the matrix constraint implies that $a_{j}=b_{j}= \pm \mathrm{i} m$ for all $j=1, \ldots, N$ while the scalar constraint implies the much weaker condition $\sum_{j=1}^{N}\left(a_{j}^{2}+b_{j}^{2}\right)=-2 \mathrm{Nm}^{2}$.

As a representative solution of the matrix constraint we have already given (4.30). Its stabiliser was determined to have the Lie algebra $\mathfrak{u}(1) \oplus \mathfrak{s u}(N) \oplus \mathfrak{s u}(N)$ and thus the size of the corresponding orbit $\mathcal{O}_{(4.30)}$ is

$$
\begin{aligned}
\operatorname{dim} \mathcal{O}_{(4.30)} & =\operatorname{dim} \mathfrak{s u}(N, N)-\operatorname{dim}(\mathfrak{u}(1) \oplus \mathfrak{s u}(N) \oplus \mathfrak{s u}(N))=(2 N)^{2}-1-\left(1+2\left(N^{2}-1\right)\right) \\
& =2 N^{2}
\end{aligned}
$$

by applying the orbit-stabiliser theorem. The same is true for any other orbit satisfying the matrix constraint (4.27).

\footnotetext{
${ }^{14}$ Note that $\mathbb{P}=\operatorname{Ad}_{b}^{*}\left(m \mathcal{P}_{0}\right)$ belongs to a 'momentum' orbit, which is different from the coadjoint orbit, $\left\{\operatorname{Ad}_{g}^{*}\left(m \mathcal{P}_{0}\right) \mid \forall g \in \mathrm{cPoin}_{3}(N)\right\}$, of coloured Poincaré symmetry $\mathrm{cPoin}_{3}(N)$. The coadjoint orbit can be viewed as the 'phase space', and hence the momentum orbit is its Lagrangian subspace. Because the (coloured) Poincaré symmetry is not semi-simple, the coadjoint orbits are not isomorphic to the adjoint orbits. In fact the momentum orbit can be viewed as either the adjoint orbit of $\mathrm{cPoin}_{3}(N)$ or the coadjoint orbit of the coloured Lorentz group $\mathrm{SU}(N, N)$.
} 
By contrast, if only the scalar constraint (4.23) is satisfied, there are many more solutions. Consider for example

$$
\mathbb{P}=\sqrt{N} \operatorname{diag}(\mathrm{i} m, 0,0, \ldots, 0,-\mathrm{i} m),
$$

which solves the trace but not the matrix constraint. The stabiliser is in this case generated by $\mathfrak{u}(1) \oplus \mathfrak{u}(N-1, N-1)$, so that the orbit is of dimension

$$
\operatorname{dim} \mathcal{O}_{(4.41)}=\operatorname{dim} \mathfrak{s u}(N, N)-\operatorname{dim}(\mathfrak{u}(1) \oplus \mathfrak{u}(N-1, N-1))=8 N-6
$$

and is a smaller orbit than the one dictated by the matrix constraint for $N>3$.

As a final example we consider

$$
\mathbb{P}=\sqrt{\frac{6}{(2 N+1)(N+1)}} \operatorname{diag}(\mathrm{i} m, 2 \mathrm{i} m, \ldots, N \mathrm{i} m,-N \mathrm{i} m, \ldots,-2 \mathrm{i} m,-\mathrm{i} m),
$$

which solves the trace but not the matrix constraint. Its stabiliser is $\mathfrak{u}(1)^{2 N-1}$ and the orbit has dimension

$$
\operatorname{dim} \mathcal{O}_{(4.43)}=\operatorname{dim} \mathfrak{s u}(N, N)-\operatorname{dim}\left(\mathfrak{u}(1)^{2 N-1}\right)=2 N(2 N-1) .
$$

This is the maximal dimension of a semi-simple orbit since the stabiliser is the minimal one: just the Cartan subalgebra. The dimension of the orbit equals the number of roots of $\mathfrak{s u}(N, N)$ and is much larger than the orbits corresponding to the matrix constraint for $N>1$.

Generally, (complex) semi-simple orbits are related to representative elements in the closure of the fundamental Weyl chamber (within the Cartan subalgebra) [58, §2]. Fully generic elements in the interior of the fundamental Weyl chamber correspond to elements such as the example (4.43) and the corresponding semi-simple orbits are of maximal dimension. If the semi-simple element is non-generic and has a larger stabiliser, it lies on boundaries of the fundamental Weyl chamber and the dimension of the orbit shrinks. The matrix constraint (4.27) selects a very specific set of such boundaries where all orbits have the same dimension $(2 N)^{2}$. As is clear from the examples above, the trace constraint (4.23) allows for a wide variety of orbits. In fact, from a projective point of view all possible semisimple orbits are allowed.

Thinking of different semi-simple orbits as different types of massive particles, just like positive and negative energy particles in the uncoloured case, we conclude that the geometric action (4.21) and the canonical action (4.25) contain all possible types of massive particles, where even the number of degrees of freedom can change.

By contrast, the canonical action (4.26) only contains massive particles with $(2 N)^{2}$ degrees of freedom and contains all orbits of this dimension.

The orbit action (4.28) describes only a single orbit and the choice of a type of massive particle corresponds to a choice of semi-simple orbit.

For completeness, we give the general form of all representatives of solutions of the matrix constraint (4.27). By using $\mathrm{SU}(N, N)$ transformations, the diagonal solution for 
$\mathbb{P}^{2}+m^{2} \llbracket=0$ can be brought into the form

$$
\widehat{\mathbb{P}}_{N-2 \ell}=m \operatorname{diag}(\underbrace{\mathrm{i}, \ldots, \mathrm{i}}_{\ell}, \underbrace{-\mathrm{i}, \ldots,-\mathrm{i}}_{N-\ell}, \underbrace{\mathrm{i}, \ldots \mathrm{i}}_{N-\ell}, \underbrace{-\mathrm{i}, \ldots,-\mathrm{i}}_{\ell}),
$$

where we have taken into account the equivalence of interchanging diagonal elements among the first and the second $N$ entries respectively, since such interchanges can be made by an $\mathrm{SU}(N, N)$ action. Here, the number $\ell$ is the number of the element $+\mathrm{i}$ in the first $N$ entries. Hence, $\ell$ can take $N+1$ possible values $(\ell=0,1, \ldots, N)$ and there exist $N+1$ distinct orbits satisfying the equation $\mathbb{P}^{2}+m^{2} \rrbracket=0$. The dimension of the orbit $\mathcal{O}_{N-2 \ell}$ generated by $\widehat{\mathbb{P}}_{N-2 \ell}$ is simply $2 N^{2}$, and it corresponds to the homogeneous space,

$$
\mathcal{O}_{N-2 \ell} \simeq \mathrm{U}(N, N) /(\mathrm{U}(N-\ell, \ell) \times \mathrm{U}(N-\ell, \ell)),
$$

which is a pseudo-Euclidean flag variety. The stabiliser is compact only for $\ell=0$ and $\ell=N$. Note that the dimension does not depend on $\ell$. As mentioned previously, the orbits that we describe here correspond to semi-simple coadjoint orbits of $\mathfrak{s u}(N, N)$ (and they are related by Weyl reflections).

\subsubsection{Massless particles: nilpotent orbits}

For the case $m=0$, both the matrix and the trace constraint require the orbit to have a nilpotent representative. Nilpotent orbits of $\mathrm{SU}(N, N)$ are described by signed Young tableaux [58, Thm. 9.3.3]. These are Young diagrams with $2 N$ boxes filled with $N$ positive and $N$ negative signs in such a way that signs alternate across rows. Two signed Young diagrams are regarded as equivalent if and only if one can be obtained from the other by interchanging rows of equal length. Say, the height of the $k$-th column is $h_{k}$, then the dimension of the orbit corresponding to the Young diagram $\lambda=\left[h_{1}, \ldots, h_{2 N}\right]$ is

$$
\operatorname{dim} \mathcal{O}_{\lambda}=4 N^{2}-\sum_{k=1}^{2 N} h_{k}^{2} .
$$

The orbits with dimension $2 N^{2}$ correspond to the Young diagram $\lambda=[N, N]$ and there are $N+1$ different signed Young diagrams:

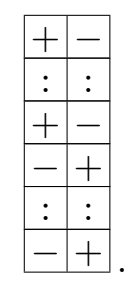

These are the nilpotent orbits which are the $m \rightarrow 0$ limits of the semi-simple orbits described by the matrix constraint $\mathbb{P}^{2}=-m^{2} \rrbracket$, and they obviously satisfy the orbit equation $\mathbb{P}^{2}=0$. However, the algebraic variety given by the latter equation does not contain only the $2 N^{2}$ dimensional nilpotent orbits, but also other nilpotent orbits of smaller dimensions. Such orbits correspond to all signed Young diagrams of the type $\boldsymbol{\lambda}=[2 N-k, k]$ 
(which can be obtained from the Young diagram (4.48) by moving boxes from the second to the first column). The number of such signed Young diagrams is $k+1$ and its dimension is $4 N^{2}-(2 N-k)^{2}-k^{2}=4 N k-2 k^{2}$. The number $k$ takes value in $\{0, \ldots, N\}$ including the $2 N^{2}$ dimensional one with $k=N$, so the equation $\mathbb{P}^{2}=0$ describes $\sum_{k=0}^{N}(k+1)=(N+1)(N+2) / 2$ distinct orbits including the trivial one $\mathbb{P}=0$. The massless particles associated to these orbits are discussed in section 4.7 .

\subsection{Massive coloured particle in component form}

We now work out the Lagrangians in more detail, beginning with the more general one (4.26) that contains all massive orbits satisfying the matrix-valued 'mass-shell' constraint (4.27).

Plugging the parametrisations (4.5), (4.9) and (4.12) into the action (4.26) leads to the Lagrangian

$$
L=\frac{1}{N} \operatorname{tr}\left[\dot{\mathbf{X}}^{a} \mathbf{P}_{a}-\dot{\mathbf{Y}} \boldsymbol{\Pi}+\boldsymbol{\Lambda}\left(\mathbf{P}_{a} \mathbf{P}^{a}-\boldsymbol{\Pi}^{2}+m^{2} \mathbf{I}\right)+\mathbf{K}_{a}\left(\left\{\mathbf{P}^{a}, \boldsymbol{\Pi}\right\}+\frac{\mathrm{i}}{2} \varepsilon^{a b c}\left[\mathbf{P}_{b}, \mathbf{P}_{c}\right]\right)\right],
$$

where our space-time conventions were given in footnote 5 .

Using the parametrisations (4.6), (4.10) and (4.13), the Lagrangian (4.49) becomes

$$
\begin{aligned}
L= & \dot{x}^{a} p_{a}+\frac{1}{N} \dot{x}_{I}^{a} p_{a}^{I}-\frac{1}{N} \dot{y}^{I} \pi_{I}+e\left(p^{a} p_{a}+\frac{1}{N} p_{I}^{a} p_{a I}-\frac{1}{N} \pi^{I} \pi_{I}+m^{2}\right) \\
& +\frac{1}{N} \lambda_{I}\left(2 p^{a} p_{a}^{I}-\frac{1}{2} d^{J K I} p_{J}^{a} p_{a K}-\frac{1}{2} d^{J K I} \pi_{J} \pi_{K}\right) \\
& +\frac{2}{N} f_{a} p_{I}^{a} \pi^{I}+\frac{1}{N} k_{a I}\left(2 p^{a} \pi^{I}-d^{J K I} p_{a J} \pi_{K}+\frac{1}{2} \varepsilon^{a b c} f^{J K I} p_{b J} p_{c K}\right),
\end{aligned}
$$

when we also use the algebra (2.1). The adjoint $\mathfrak{s u}(N)$ indices $I$ are raised and lowered with the invariant $\delta_{I J}$.

Focussing on the terms that do not depend on $N$, we recognise immediately the usual uncoloured (4.16). The terms proportional to $1 / N$ represent the effects of colouring the Poincaré algebra.

We record the equations of motion implied by (4.50)

$$
\begin{aligned}
\dot{p}_{a}=0, \quad \dot{p}_{a}^{I}=0, \quad \dot{\pi}_{I} & =0, \\
\dot{x}^{a}+2 e p^{a}+\frac{2}{N} \lambda_{I} p^{a I}+\frac{2}{N} k_{a I} \pi^{I} & =0, \\
\dot{x}_{I}^{a}+2 e p_{I}^{a}+2 \lambda_{I} p^{a}-d^{I J K} \lambda_{J} p_{a K}+2 f_{a} \pi^{I}-d^{I J K} k_{a J} \pi_{K}+\varepsilon_{a b c} f^{I J K} k_{J}^{b} p_{K}^{c} & =0, \\
-\dot{y}^{I}-2 e \pi^{I}-d^{I J K} \pi_{J} \pi_{K}+2 f_{a} p^{a I}+2 k_{a}^{I} p^{a}-d^{I J K} k_{a J} p_{K}^{a} & =0, \\
p^{a} p_{a}+m^{2}+\frac{1}{N} p_{I}^{a} p_{a}^{I}-\frac{1}{N} \pi^{I} \pi_{I} & =0, \\
2 p_{a}^{I} p^{a}-\frac{1}{2} d^{I J K} p_{a J} p_{K}^{a}-\frac{1}{2} d^{I J K} \pi_{J} \pi_{K} & =0, \\
p_{a}^{I} \pi_{I} & =0, \\
2 p^{a} \pi^{I}-d^{I J K} p_{J}^{a} \pi_{K}+\frac{1}{2} \varepsilon^{a b c} f^{I J K} p_{b J} p_{c K} & =0 .
\end{aligned}
$$


The constraints that are enforced by the Lagrange multipliers are given in (4.51e)(4.51h). The total number of constraints is $1+\left(N^{2}-1\right)+3+3\left(N^{2}-1\right)=(2 N)^{2}$ since the Lagrange multiplier $\mathbb{L}$ belongs to $\mathfrak{u}(N, N)$. We note that these constraints are not all independent and we are dealing with a reducible system of constraints. This will be further investigated in section 4.5.

While the Lagrangian (4.50) is valid for any choice of $\mathbb{P}$ satisfying $\mathbb{P}^{2}=-m^{2} \llbracket$, the orbit Lagrangian (4.28) depends on the choice of a given orbit where this condition is satisfied. In order to investigate this we take a representative momentum and repeat the analysis from appendix B in connection with the non-linear realisation discussed above.

Written in matrix form, the representative momentum (4.30) takes the form

$$
\widehat{\mathbb{P}}=m\left(\begin{array}{cc}
\mathrm{i} \mathbf{I} & 0 \\
0 & -\mathrm{i} \mathbf{I}
\end{array}\right)
$$

while the boost element $b$ from (4.33b) can be written as

$$
b=\exp \left[\frac{1}{2}\left(\begin{array}{cc}
0 & \mathbf{V}^{+} \\
\mathbf{V}^{-} & 0
\end{array}\right)\right], \quad \text { with } \quad\left(\mathbf{V}^{+}\right)^{\dagger}=\mathbf{V}^{-} .
$$

Other elements of $\mathfrak{s u}(N, N)$ stabilise $\widehat{\mathbb{P}}$ under the adjoint action and belong to the subalgebra $\mathfrak{u}(1) \oplus \mathfrak{s u}(N) \oplus \mathfrak{s u}(N)$.

The orbit of the reference momentum is then given by

$$
b \widehat{\mathbb{P}} b^{-1}=m\left(\begin{array}{l}
\mathrm{i} \cosh \sqrt{\mathbf{V}^{+} \mathbf{V}^{-}}-\mathrm{i} \frac{\sinh \sqrt{\mathbf{V}^{+} \mathbf{V}^{-}}}{\sqrt{\mathbf{V}^{+} \mathbf{V}^{-}}} \mathbf{V}^{+} \\
\mathrm{i} \mathbf{V}^{-} \frac{\sinh \sqrt{\mathbf{V}^{+} \mathbf{V}^{-}}}{\sqrt{\mathbf{V}^{+} \mathbf{V}^{-}}}-\mathrm{i} \cosh \sqrt{\mathbf{V}^{-} \mathbf{V}^{+}}
\end{array}\right),
$$

where the hyperbolic trigonometric functions of the hermitian matrices $\mathbf{V}^{+} \mathbf{V}^{-}$and $\mathbf{V}^{-} \mathbf{V}^{+}$ are defined by their power series expansions. The formula represents the action of a general coloured Lorentz boost on the reference momentum (4.52). We rewrite this more compactly by defining

$$
\mathbf{P}^{+}=-m \mathrm{i} \frac{\sinh \sqrt{\mathbf{V}^{+} \mathbf{V}^{-}}}{\sqrt{\mathbf{V}^{+} \mathbf{V}^{-}}} \mathbf{V}^{+}, \quad \mathbf{P}^{-}=\left(\mathbf{P}^{+}\right)^{\dagger},
$$

leading to

$$
b \widehat{\mathbb{P}} b^{-1}=\left(\begin{array}{cc}
\mathrm{i} \sqrt{m^{2} \mathbf{I}+\mathbf{P}^{+} \mathbf{P}^{-}} & \mathbf{P}^{+} \\
\mathbf{P}^{-} & -\mathrm{i} \sqrt{m^{2} \mathbf{I}+\mathbf{P}^{-} \mathbf{P}^{+}}
\end{array}\right),
$$

where the square root of matrices is again defined by its Taylor series around $\mathbf{P}^{ \pm} \mathbf{P}^{\mp}=0$. We note that all elements in the orbit of the reference momentum (4.52) satisfy

$$
\left(b \widehat{\mathbb{P}} b^{-1}\right)^{2}=\widehat{\mathbb{P}}^{2}=-m^{2} \square .
$$

This equation has more solutions than (4.56) and the Lagrangian (4.26) captures all of them. 
Considering the Lagrangian (4.28) for the reference momentum (4.52) we need to pair (4.56) with the velocity vector $\dot{\mathbb{X}}$ given in (4.5), which leads to

$$
L_{\text {orb }}=\frac{1}{2 N} \operatorname{tr}\left[\left(\dot{\mathbf{X}}_{0}-\dot{\mathbf{Y}}\right) \sqrt{m^{2} \mathbf{I}+\mathbf{P}^{+} \mathbf{P}^{-}}+\left(\dot{\mathbf{X}}_{0}+\dot{\mathbf{Y}}\right) \sqrt{m^{2} \mathbf{I}+\mathbf{P}^{-} \mathbf{P}^{+}}+\dot{\mathbf{X}}^{+} \mathbf{P}^{-}+\dot{\mathbf{X}}^{-} \mathbf{P}^{+}\right] .
$$

This is the coloured generalisation of the Lagrangian (4.20). Setting $N=1$ and $\dot{\mathbf{X}}^{a}, \mathbf{P}^{a}$ to 3-component Lorentz vectors and $\dot{\mathbf{Y}}=0$ reduces it manifestly to the standard uncoloured massive particle.

The Lagrangian (4.58) has been deduced from the representative momentum (4.52). We shall now explore the relation to the previously derived Lagrangian (4.49) and try to follow a route similar to that of appendix B that starts from the constraints implied by (4.58). Varying (4.58) with respect to $\mathbf{X}^{0} \pm \mathbf{Y}$ leads to the constraints

$$
\mathbf{C}_{ \pm}=\mathbf{P}^{0} \pm \mathbf{\Pi}-\sqrt{m^{2} \mathbf{I}+\mathbf{P}^{ \pm} \mathbf{P}^{\mp}}=0
$$

that are $(N \times N)$-matrix valued and where we have introduced new $(N \times N)$-matrix valued variables $\mathbf{P}^{0}=-\mathbf{P}_{0}$ and $\boldsymbol{\Pi}$ as integration constants. These constraints are the generalisations of the positive-energy mass-shell constraint of an uncoloured particle as in (4.20). In order to allow for generalisations of negative energy, we shall consider as a generalisation the constraints

$$
\mathbf{C}_{ \pm}^{2}=\left(\mathbf{P}^{0} \pm \mathbf{\Pi}\right)^{2}-\left(m^{2} \mathbf{I}+\mathbf{P}^{ \pm} \mathbf{P}^{\mp}\right)=0 .
$$

These are also $(N \times N)$-matrix valued and will be enforced using the matrix-valued Lagrange multipliers $\boldsymbol{\Lambda} \pm \mathbf{K}$. Enforcing only these squared constraints allows for more solutions than implied by the original Lagrangian in the same way as squaring the positive mass condition for going from (4.20) to (4.16) allows for more solutions, see also appendix B.

The resulting generalised Lagrangian is then

$$
\begin{aligned}
\tilde{L}= & \frac{1}{2 N} \operatorname{tr}\left[-\left(\dot{\mathbf{X}}^{0}+\dot{\mathbf{Y}}\right)\left(\mathbf{P}^{0}+\boldsymbol{\Pi}\right)-\left(\dot{\mathbf{X}}^{0}-\dot{\mathbf{Y}}\right)\left(\mathbf{P}^{0}-\boldsymbol{\Pi}\right)+\mathbf{P}^{+} \dot{\mathbf{X}}^{-}+\mathbf{P}^{-} \dot{\mathbf{X}}^{+}\right. \\
& \left.-(\boldsymbol{\Lambda}+\mathbf{K})\left(\left(\mathbf{P}^{0}+\boldsymbol{\Pi}\right)^{2}-\left(m^{2} \mathbf{I}+\mathbf{P}^{+} \mathbf{P}^{-}\right)\right)-(\boldsymbol{\Lambda}-\mathbf{K})\left(\left(\mathbf{P}^{0}-\boldsymbol{\Pi}\right)^{2}-\left(m^{2} \mathbf{I}+\mathbf{P}^{-} \mathbf{P}^{+}\right)\right)\right] \\
= & \frac{1}{N} \operatorname{tr}\left[\dot{\mathbf{X}}^{a} \mathbf{P}_{a}-\dot{\mathbf{Y}} \boldsymbol{\Pi}+\boldsymbol{\Lambda}\left(\mathbf{P}^{a} \mathbf{P}_{a}-\mathbf{\Pi}^{2}+m^{2} \mathbf{I}\right)-\mathbf{K}\left(\left\{\mathbf{P}^{0}, \boldsymbol{\Pi}\right\}+\mathrm{i}\left[\mathbf{P}^{1}, \mathbf{P}^{2}\right]\right)\right]
\end{aligned}
$$

with $\mathbf{P}^{ \pm}=\mathbf{P}_{1} \pm \mathrm{i} \mathbf{P}_{2}=\mathbf{P}^{1} \pm \mathrm{i} \mathbf{P}^{2}$ as well as $\mathbf{P}_{0}=-\mathbf{P}^{0}$ due to the $(-++)$ signature.

The Lagrangian (4.61) does not manifestly agree with (4.49) that we deduced from the natural squared constraint (4.27) in $\mathfrak{u}(N, N)$, in particular, it does not exhibit manifest Lorentz invariance under the usual $\mathrm{SO}(1,2)$ group. However, the transformation of the Lagrange multipliers under the coloured Lorentz group is exactly such that it is opposite to that of the constraints so that the Lagrangian is invariant by construction.

The matrix mass-shell Lagrangian (4.49) also has two extra Lagrange multipliers $\mathbf{K}_{1}$ and $\mathbf{K}_{2}$ that are not present in (4.61). The constraints they enforce from (4.49) are

$$
\left\{\mathbf{P}^{1}, \boldsymbol{\Pi}\right\}+\mathrm{i}\left[\mathbf{P}_{2}, \mathbf{P}_{0}\right]=0, \quad\left\{\mathbf{P}^{2}, \boldsymbol{\Pi}\right\}+\mathrm{i}\left[\mathbf{P}_{0}, \mathbf{P}_{1}\right]=0
$$


that are equivalent to

$$
\left(\mathbf{P}_{0} \pm \Pi\right) \mathbf{P}^{ \pm}=\mathbf{P}^{ \pm}\left(\mathbf{P}_{0} \mp \boldsymbol{\Pi}\right) .
$$

When the original constraint (4.59) is satisfied, meaning $\mathbf{P}_{0} \pm \mathbf{\Pi}=\sqrt{m^{2} \mathbf{I}+\mathbf{P}^{\mp} \mathbf{P}^{ \pm}}$, the above constraints follow. However, they need not follow from the squared constraint $\mathbf{C}_{ \pm}^{2}=0$ in (4.60) and thus the manifestly covariant Lagrangian (4.49) is not equivalent to (4.61). We therefore consider the manifestly covariant Lagrangian (4.49) as the appropriate generalisation of the massive particle (4.52) to all possible massive orbits and shall work with (4.49) in the following.

\subsection{Constraint structure and degrees of freedom}

In this section we revisit the discussion of the dimension of semi-simple orbits of section 4.3 and relate it to the reducibility of the constraints implied by the matrix constraint (4.27). The reducibility of the constraint means that not all of the $(2 N)^{2}$ components of $(4.27)$ can be counted independently. Rather, we are interested in the dimension of the solution

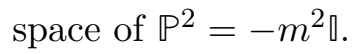

From the discussion in section 4.3 we know that the dimension of the solution space is given by the size of an orbit of solutions and that these can be characterised by the stabilisers of the semi-simple representative of the orbit. For the case of the massive momentum given in (4.30) the stabiliser was $S(\mathrm{U}(N) \times \mathrm{U}(N)) \subset \mathrm{SU}(N, N)$. For any other orbit of solutions of (4.27) the stabiliser is a similar real form of the same dimension. The dimension of the stabiliser equals the number of independent constraints contained in (4.27) since this is the number by which the number of variables is reduced to obtain an orbit of solutions.

A canonical analysis then reveals the number of degrees of freedom. It is important to consider the configuration space variables of $(4.26)$ as $\mathbb{X}, \mathbb{P}$ and $\mathbb{L}$, where the first two have $(2 N)^{2}-1$ components while $\mathbb{L}$ has $(2 N)^{2}$ components. Canonically, the conjugate momenta of $\mathbb{P}$ and $\mathbb{L}$ vanish and there is another constraint relating the conjugate momentum of $\mathbb{X}$ to $\mathbb{P}$. These are all the primary constraints and their numbers are $(2 N)^{2}-1,(2 N)^{2}$ and $(2 N)^{2}-1$, respectively.

The constraint (4.27) appears as a secondary constraint after time evolution of the primary constraint that the momentum $\pi_{\mathbb{L}}$ conjugate to $\mathbb{L}$ vanishes. As we argued above, there are only $2 N^{2}-1$ independent constraints contained in (4.27) in agreement with the dimension of the stabiliser of a solution. These $2 N^{2}-1$ constraints and the $(2 N)^{2}$ conditions $\pi_{\mathbb{L}}=0$ are first-class constraints, the remaining two sets of constraints are second-class. The count of degrees of freedom in phase space is therefore

$$
\underbrace{2 \times\left((2 N)^{2}-1\right)}_{\left(\mathbb{K}, \pi_{\mathfrak{X}}\right)}+\underbrace{2 \times\left((2 N)^{2}-1\right)}_{\left(\mathbb{P}, \pi_{\mathbb{P}}\right)}+\underbrace{2 \times(2 N)^{2}}_{\left(\mathbb{L}, \pi_{\mathbb{L}}\right)}-2 \underbrace{\left((2 N)^{2}+2 N^{2}-1\right)}_{\text {first-class }}-\underbrace{2\left((2 N)^{2}-1\right)}_{\text {second-class }}=2 \times 2 N^{2} .
$$

Since this counting is in phase space, the number of degrees of freedom in configuration space is $2 N^{2}$ which agrees with the dimension of the orbit momentum: $(2 N)^{2}-1-\left(2 N^{2}-\right.$ 1) $=2 N^{2}$ studied in section 4.3 . 
Let us also compare the Hamiltonian action (4.26) that contains the matrix Lagrange multiplier to the geometric action (4.21).

The equations of motion following from the Hamiltonian action (4.26) written in matrix form are

$$
\begin{aligned}
\dot{\mathbb{P}} & =0, \\
\dot{\boldsymbol{\chi}}+\mathbb{P} \mathbb{L}+\mathbb{Q} \mathbb{P} & =0, \\
\mathbb{P}^{2}+m^{2} \llbracket & =0 .
\end{aligned}
$$

The derivative here is with respect to the Lagrangian time variable. The second equation (4.66) is uniquely solvable for $\mathbb{P}$ for generic $\mathbb{L}$; a closed form expression is not available but a formal series expansion can be obtained. Instead of solving for $\mathbb{P}$ we verify that the equations of motion agree with those of the geometric action (4.21). We deduce from (4.66)

$$
\dot{\mathbb{X}}+\frac{1}{m^{2}} \mathbb{P} \dot{X} \mathbb{P}=0
$$

and since $\mathbb{P}$ is constant, the same equation also holds for $\ddot{\mathbb{X}}$

$$
\ddot{\mathbb{X}}+\frac{1}{m^{2}} \mathbb{P} \ddot{X} \mathbb{P}=0 .
$$

This equation can be understood as the projection of $\ddot{\mathbb{X}}$ to what in the uncoloured case would be the transversal directions to $\mathbb{P}$. If we can also argue that the 'longitudinal' components

$$
\ddot{\mathbb{X}}-\frac{1}{m^{2}} \mathbb{P} \ddot{\mathbb{X}} \mathbb{P}
$$

vanish, then the Hamiltonian equations would imply $\ddot{\mathbb{K}}=0$ in agreement with the equations of the geometric Lagrangian (4.21) (in proper time gauge). In the uncoloured case the longitudinal combinations are gauge-variant and can be set to zero by a choice of gauge and for this reason we now study the gauge symmetries of the system (4.26).

The gauge transformations entailed by the matrix constraint (4.67) are for matrix parameter $\mathrm{i} \mathbb{E} \in \mathfrak{u}(N, N)$

$$
\delta_{\mathbb{E}} \mathbb{P}=0, \quad \delta_{\mathbb{E}} \mathcal{X}=\{\mathbb{E}, \mathbb{P}\}, \quad \delta_{\mathbb{E}} \mathbb{L}=-\dot{\mathbb{E}} .
$$

These gauge transformations are reducible since $\mathbb{X}$ is left invariant by any gauge parameter of the form $\mathbb{E}=[\mathbb{F}, \mathbb{P}]$ for an arbitrary matrix $\mathbb{E}$ when $(4.67)$ is used. If this $\mathbb{E}$ is itself of the form $\{\mathbb{G}, \mathbb{P}\}$, it leads to a vanishing $\mathbb{E}$ when (4.67) is used. This reflects the fact that the constraints form a reducible system of gauge transformations. Intuitively, the total effective number of gauge parameters is given by $(2 N)^{2}\left[\sum_{n=0}^{\infty}(-1)^{n}\right]-1=2 N^{2}-1$ by geometric sum regularisation and where the -1 is due to the identity component that has to be treated differently. The reducibility of the gauge transformations is tied to the reducibility of the constraint (4.67) 
The longitudinal component (4.70) transforms as follows under gauge transformations

$$
\delta_{\mathbb{E}}\left[\ddot{\mathbb{K}}-\frac{1}{m^{2}} \mathbb{P} \ddot{\mathbb{K}} \mathbb{P}\right]=2\{\ddot{\mathbb{E}}, \mathbb{P}\}
$$

and is clearly gauge-variant. Moreover, it is in the kernel of the 'transversal' projection (4.68). We therefore expect to be able to gauge the longitudinal part to zero fully. For this to be correct, we need that any element in the kernel of the transversal projection is of the from $\{\ddot{\mathbb{E}}, \mathbb{P}\}$ for some $\ddot{\mathbb{E}}$ with $\mathbb{P}$ satisfying (4.67). This can be checked to be true.

We therefore conclude that the geometric action (4.21) and the Hamiltonian action (4.26) with matrix constraint both imply the equation of motion

$$
\ddot{x}=0
$$

upon fully fixing the gauge invariances. In the geometric case this only requires the choice of proper time gauge $\frac{1}{2 N} \operatorname{Tr}\left[\dot{K}^{2}\right]=-1$ while for the Hamiltonian action this requires fixing all longitudinal components (4.70) to zero which is a larger set of gauge constraints. The two systems are therefore not equivalent and this agrees also with the analysis of which orbits they support that we carried out in section 4.3.

\subsection{Examples for small $N$}

We shall now analyse the Lagrangian (4.49) for small values of $N$, beginning with the uncoloured $N=1$ case as a quick consistency check. It is useful to start with the component expression (4.50).

The uncoloured $N=1$ case. For the case $N=1$, there are no $\mathfrak{s u}(N)$ generators $T^{I}$ and the symmetry group is just the usual Poincaré group in $2+1$ dimensions. Without $T^{I}$ generators there are no coordinates $x_{I}^{a}, y^{I}$ or associated momentum or Lagrange multiplier components. The Lagrangian (4.50) reduces simply to

$$
L=\dot{x}^{a} p_{a}+e\left(p^{a} p_{a}+m^{2}\right) .
$$

This agrees with the well-known free massive particle (4.16). One can check similarly that the 'orbit Lagrangian' (4.58) reduces to (4.20).

The only Lagrange multiplier is $e$ and the corresponding scalar constraint $p^{a} p_{a}+m^{2}=0$ has two branches of solution up to Lorentz rotation of the momentum, corresponding to positive and negative energy particles.

The case $\boldsymbol{N}=\mathbf{2}$. For the case $N=2$ we can rewrite (4.50) by using $\mathfrak{s u}(2) \cong \mathfrak{s o}(3)$ to replace $\mathfrak{s u}(2)$ adjoint indices $I$ by $\mathfrak{s o}(3)$ vectors, e.g. $p_{a}^{I} \rightarrow \vec{p}_{a}$. Then the trace $\delta_{I J}$ becomes the scalar product of vectors and $f^{I J K}$ becomes the cross-product. Note that $d^{I J K}=0$ for $N=2$. We will continue to write out the $\mathfrak{s o}(1,2)$ Lorentz indices that are raised and lowered with the Minkowski metric.

Then (4.50) becomes

$$
\begin{aligned}
L= & \dot{x}^{a} p_{a}+\frac{1}{2} \dot{\vec{x}}^{a} \cdot \vec{p}_{a}-\frac{1}{2} \dot{\vec{y}} \cdot \vec{\pi}+e\left(p^{a} p_{a}+\frac{1}{2} \vec{p}^{a} \cdot \vec{p}_{a}-\frac{1}{2} \vec{\pi} \cdot \vec{\pi}+m^{2}\right)+\vec{\lambda} \cdot \vec{p}^{a} p_{a} \\
& +f_{a} \vec{p}^{a} \cdot \vec{\pi}+\frac{1}{2} \vec{k}_{a} \cdot\left(2 \vec{\pi} p^{a}+\frac{1}{2} \varepsilon^{a b c} \vec{p}_{b} \times \vec{p}_{c}\right) .
\end{aligned}
$$


The constraints implied by this Lagrangian from varying the Lagrange multipliers $e$, $\vec{\lambda}, f_{a}$ and $\vec{k}_{a}$ are

$$
\begin{aligned}
(1 \text { constraint }) & p^{a} p_{a}+\frac{1}{2} \vec{p}^{a} \cdot \vec{p}_{a}-\frac{1}{2} \vec{\pi} \cdot \vec{\pi}+m^{2}=0, \\
(3 \text { constraints }) & \vec{p}^{a} p_{a}=0, \\
(3 \text { constraints }) & \vec{p}^{a} \cdot \vec{\pi}=0, \\
(3 \times 3 \text { constraints }) & 4 \vec{\pi} p^{a}+\varepsilon^{a b c} \vec{p}_{b} \times \vec{p}_{c}=0 .
\end{aligned}
$$

We have also written out the number of equations. The total of 16 constraints is reducible. There are combinations that vanish identically without the use of the equations of motion and the simplest example is given by $\vec{\pi} \cdot\left(\vec{p}^{a} p_{a}\right)-p_{a}\left(\vec{\pi} \vec{p}^{a}\right)$ which is a single constraint. According to the general discussion in section 4.3, the solution space to the constraints is eight-dimensional for $N=2$ and we shall analyse this by solving them explicitly.

We first consider the case when the particle has non-vanishing ordinary energy, so that $p^{0} \neq 0$. Then we can use all three equations of $(4.76 \mathrm{~b})$ to solve for $\vec{p}^{0}$ by

$$
\vec{p}^{0}=\frac{1}{p^{0}}\left(\vec{p}^{1} p_{1}+\vec{p}^{2} p_{2}\right) .
$$

Similarly, the three $a=0$ components of $(4.76 \mathrm{~d})$ can be used to solve for $\vec{\pi}$ as

$$
\vec{\pi}=-\frac{1}{2 p^{0}} \vec{p}_{1} \times \vec{p}_{2}
$$

Substituting these two solutions into (4.76c) shows that they are satisfied automatically, therefore the constraints are 1-stage reducible. Moreover, substituting them into the $a=1$ and $a=2$ components of (4.76d) imposes no further constraints either, reflecting again the reducibility. The only remaining constraint is (4.76a) which yields the scalar constraint

$$
p^{a} p_{a}+m^{2}+\frac{1}{2 p^{0} p_{0}}\left(\vec{p}_{1} p_{1}+\vec{p}_{2} p_{2}\right)^{2}+\frac{1}{2} \vec{p}_{1} \cdot \vec{p}_{1}+\frac{1}{2} \vec{p}_{2} \cdot \vec{p}_{2}+\frac{1}{8 p^{0} p_{0}}\left(\vec{p}_{1} \times \vec{p}_{2}\right) \cdot\left(\vec{p}_{1} \times \vec{p}_{2}\right)=0 .
$$

This is one equation for the nine variables $p^{a}, \vec{p}_{1}$ and $\vec{p}_{2}$, leading in general to an eightdimensional solution space. This can be checked explicitly for generic values by reading the above equation as a quadratic equation for $p^{0} p_{0}$ and studying its discriminant. Among the four solutions of $p_{0}= \pm \omega_{1}\left(p_{1}, p_{2}, \vec{p}_{1}, \vec{p}_{2}\right), \pm \omega_{2}\left(p_{1}, p_{2}, \vec{p}_{1}, \vec{p}_{2}\right)$ with

$$
\omega_{1}\left(p_{1}, p_{2}, \vec{p}_{1}, \vec{p}_{2}\right)>p_{1}^{2}+p_{2}^{2}+\frac{1}{2} \vec{p}_{1}^{2}+\frac{1}{2} \vec{p}_{2}^{2}+m^{2}>\omega_{2}\left(p_{1}, p_{2}, \vec{p}_{1}, \vec{p}_{2}\right)>0 .
$$

The two surfaces $p_{0}= \pm \omega_{1}\left(p_{1}, p_{2}, \vec{p}_{1}, \vec{p}_{2}\right)$ correspond to the "momentum" shells or orbits where the energy is bounded from either below or above. These orbits, denoted by $\mathcal{O}_{+2}$ and $\mathcal{O}_{-2}$, can be viewed as the colour extension of the positive and negative energy momentum shells. The other two surfaces $p_{0}= \pm \omega_{2}\left(p_{1}, p_{2}, \vec{p}_{1}, \vec{p}_{2}\right)$ rejoin at the "equator" with $p_{0}=0,{ }^{15}$

\footnotetext{
${ }^{15}$ By solving the system (4.76) with $p_{0}=0$, one can obtain a seven dimensional surface corresponding to this "equator".
} 
and form a single orbit, denoted by $\mathcal{O}_{0}$. The orbits $\mathcal{O}_{+2}, \mathcal{O}_{0}$ and $\mathcal{O}_{-2}$ can be obtained from the representative momenta,

$$
\widehat{\mathbb{P}}_{+2}=m\left(\begin{array}{cccc}
\mathrm{i} & 0 & 0 & 0 \\
0 & \mathrm{i} & 0 & 0 \\
0 & 0 & -\mathrm{i} & 0 \\
0 & 0 & 0 & -\mathrm{i}
\end{array}\right), \quad \widehat{\mathbb{P}}_{0}=m\left(\begin{array}{cccc}
\mathrm{i} & 0 & 0 & 0 \\
0 & -\mathrm{i} & 0 & 0 \\
0 & 0 & \mathrm{i} & 0 \\
0 & 0 & 0 & -\mathrm{i}
\end{array}\right), \quad \widehat{\mathbb{P}}_{-2}=m\left(\begin{array}{cccc}
-\mathrm{i} & 0 & 0 & 0 \\
0 & -\mathrm{i} & 0 & 0 \\
0 & 0 & \mathrm{i} & 0 \\
0 & 0 & 0 & \mathrm{i}
\end{array}\right),
$$

and they correspond to the homogeneous spaces,

$$
\mathcal{O}_{ \pm 2} \simeq \mathrm{U}(2,2) /(\mathrm{U}(2) \times \mathrm{U}(2)), \quad \mathcal{O}_{0} \simeq \mathrm{U}(2,2) /(\mathrm{U}(1,1) \times \mathrm{U}(1,1)) .
$$

The first orbit $\widehat{\mathbb{P}}_{+2}$ is exactly the one analysed in (4.56) for general $N$ and the representative momentum (4.30). From (4.56) one can see that $p^{0}$ is bounded from below. The representative $\widehat{\mathbb{P}}_{-2}$ of the third orbit is the negative of $\widehat{\mathbb{P}}_{+2}$ and its orbit therefore has $p^{0}$ bounded from above. The middle orbit has points with $p^{0}=0$ and is a genuine new type of orbit for coloured Poincaré symmetry.

To recapitulate, the "momentum" space of the massive $N=2$ coloured particle consists of three distinct orbits of dimension eight, in agreement with the general discussion in section 4.3.

\subsection{Massless coloured particle}

We now consider the massless particle. Again, we make the simplest choice for its representative 'momentum' assigning zero eigenvalues to all colour associated 'momenta':

$$
P_{0}=P_{1}=E, \quad P_{2}=0, \quad P_{a}^{I}=0, \quad Q^{I}=0,
$$

where $E$ is the energy of this reference 'momentum' state. The little group is generated by $M_{0}+M_{1}, N_{0}^{I}+N_{1}^{I}$ and $T^{I}$, hence, the remaining 'broken' generators of the coloured Lorentz $\mathfrak{s u}(N, N)$ are

$$
M_{0}-M_{1}, \quad M_{2}, \quad M_{0}^{I}-M_{1}^{I}, \quad M_{2}^{I} .
$$

Again the eigenvalues (4.83) define an element $\mathcal{K}$ in the coadjoint space $\mathfrak{s u}(N, N)^{*}$ :

$$
\left\langle P_{a}, \mathcal{K}\right\rangle=E\left(\delta_{a}^{0}+\delta_{a}^{1}\right), \quad\left\langle P_{a}^{I}, \mathcal{K}\right\rangle=0, \quad\left\langle Q^{I}, \mathcal{K}\right\rangle=0 .
$$

Through the isomorphism (4.29), $\mathcal{K}$ is mapped to

$$
H_{\mathcal{K}}=E\left(P_{0}+P_{1}\right) .
$$

The massless orbit Lagrangian is given by

$$
L_{\text {orb }}=\kappa\left\langle\dot{\boldsymbol{K}}, \operatorname{Ad}_{b}^{*} \mathcal{K}\right\rangle=\frac{\kappa}{2 N} \operatorname{Tr}\left[\dot{\mathfrak{K}} b E\left(P_{0}+P_{1}\right) b^{-1}\right],
$$

where $b$ is the exponentiation of a linear combination of the generators in (4.84):

$$
b=\exp (\mathbb{V}), \quad \mathbb{V}=\left(\begin{array}{cc}
\mathbf{V} & -\mathbf{V}+\mathrm{i} \mathbf{W} \\
\mathbf{V}+\mathrm{i} \mathbf{W} & -\mathbf{V}
\end{array}\right) .
$$


Here, we packaged arbitrary linear combinations of the generators in (4.84) into $N \times N$ anti-hermitian matrices $\mathbf{V}$ and $\mathbf{W}$. The parameter $\kappa$ in (4.87) is a dimensionful scale that has no further role at the classical level.

We first notice that

$$
b E\left(P_{0}+P_{1}\right) b^{-1}=\mathbb{R} \mathbb{R}^{\dagger}\left(\begin{array}{cc}
\mathbf{I} & \mathbf{0} \\
\mathbf{0} & -\mathbf{I}
\end{array}\right),
$$

where $\mathbb{R}$ is an $N \times 2 N$ rectangular matrix,

$$
\mathbb{R}=\sqrt{E} \exp (\mathbb{V})\left(\begin{array}{c}
\mathbf{I} \\
-\mathbf{I}
\end{array}\right) .
$$

Since $\mathbb{V}$ can be decomposed as $\mathbb{V}=\mathbf{V} \otimes A+\mathrm{i} \mathbf{W} \otimes B$ and where

$$
A=\left(\begin{array}{ll}
1 & -1 \\
1 & -1
\end{array}\right), \quad B=\left(\begin{array}{ll}
0 & 1 \\
1 & 0
\end{array}\right)
$$

satisfy

$$
A^{2}=0, \quad B^{2}=I, \quad A B=-A, \quad B A=A,
$$

we find

$$
\begin{aligned}
\exp (\mathbb{V}) & =e^{\mathrm{i} \mathbf{W} \otimes B}+\int_{0}^{1} d t e^{\mathrm{i} t \mathbf{W} \otimes B}(\mathbf{V} \otimes A) e^{\mathrm{i}(1-t) \mathbf{W} \otimes B} \\
& =\cos (\mathbf{W}) \otimes I+\mathrm{i} \sin (\mathbf{W}) \otimes B+\int_{0}^{1} d t e^{\mathrm{i} t \mathbf{W} \otimes I}(\mathbf{V} \otimes A) e^{-\mathrm{i}(1-t) \mathbf{W} \otimes I} .
\end{aligned}
$$

From the above, the matrix $\mathbb{R}$ is obtained as

$$
\mathbb{R}=\left(\begin{array}{c}
\mathbf{R}+\mathrm{i} \mathbf{S} \mathbf{R} \\
-\mathbf{R}+\mathrm{i} \mathbf{S} \mathbf{R}
\end{array}\right)=\left(\begin{array}{c}
\mathbf{I}+\mathrm{i} \mathbf{S} \\
-(\mathbf{I}-\mathrm{i} \mathbf{S})
\end{array}\right) \mathbf{R}
$$

with two $N \times N$ hermitian matrices $\mathbf{R}$ and $\mathbf{S}$,

$$
\mathbf{R}=\sqrt{E} e^{-\mathrm{i} \mathbf{W}}, \quad \mathbf{S}=-2 \mathrm{i} \int_{0}^{1} d t e^{\mathrm{i} t \mathbf{W}} \mathbf{V} e^{\mathrm{i} t \mathbf{W}} .
$$

In terms of $\mathbf{R}$ and $\mathbf{S}$, the orbit is parametrised by

$$
\mathbb{P}=b E\left(P_{0}+P_{1}\right) b^{-1}=\left(\begin{array}{c}
\mathbf{I}+\mathrm{i} \mathbf{S} \\
-(\mathbf{I}-\mathrm{i} \mathbf{S})
\end{array}\right) \mathbf{R}^{2}(\mathbf{I}-\mathrm{i} \mathbf{S} \mathbf{I}+\mathrm{i} \mathbf{S}) .
$$

Note however that for a one-to-one correspondence between $\mathbf{R}$ and $\mathbf{W}$, the former matrix should be invertible. Foregoing the invertibility of $\mathbf{R}$, the parametrisation (4.96) contains other orbits besides the one generated by $E\left(P_{0}+P_{1}\right)$.

Now, let us look for algebraic constraints for $\mathbb{P}$, which lead to the parametrisation (4.96). From its explicit form of the parametrisation, one can verify

$$
\mathbb{P}^{2}=0,
$$

which is already obvious from its definition and the fact that $\left(P_{0}+P_{1}\right)^{2}=0$. 
A Lagrangian that contains all possible solutions of $\mathbb{P}^{2}=0$ is given by

$$
L=\frac{1}{2 N} \operatorname{Tr}\left[\dot{\boldsymbol{K}} \mathbb{P}+\mathbb{L} \mathbb{P}^{2}\right],
$$

which agrees with the Lagrangian (4.26) restricted to $m^{2}=0$. This is completely analogous to what happens in the uncoloured case.

Similarly to the massive coloured particle, the massless Lagrangian above contains several orbits, but this time the structure is richer: in the limit $m \rightarrow 0$, there appear new orbits of lower dimensions on the boundary of the $N+1$ massive orbits of dimension $2 N^{2}$. More precisely, the massive orbits develop open regions in the massless limit and the closure of these orbits contain such sub-orbits. The uncoloured analogue of this situation is as follows: the massive momentum orbit, which is an upper hyperboloid, becomes a cone in the massless limit. Since this cone is open near the origin, its closure contains the origin, which is the new (trivial) orbit.

While in the uncoloured case the new sub-orbit is only the trivial orbit, the situation is more interesting in the coloured case. Here, the sub-orbits are non-trivial and they may even contain sub-sub-orbits. The sub-sub-orbits, in turn, may contain sub-sub-sub-orbits, etc, exhibiting an interesting inclusion structure that is familiar from the study of co-adjoint nilpotent orbits of $\mathfrak{s u}(N, N)$ that can be arranged in a Hasse (or closure) diagram [58]. To quote the results directly, the massless coloured particle contains $k+1$ orbits of dimension $4 N k-2 k^{2}$ where $k$ varies from 0 to $N$. The orbits with $k=N$ are the massless limit of the massive orbits, whereas the other orbits with $k<N$ are newly appeared sub-orbits. The orbit with $k=0$ is the origin, corresponding to the zero-dimensional trivial orbit, see also section 4.3 for further discussions.

\subsection{Reductions to subspaces}

The full coloured Minkowski space (4.1) has coordinates $\mathbb{X}=\left(x^{a}, x_{I}^{a}, y_{I}\right)$ that transform under the coloured Poincaré algebra according to (4.4). We now consider the restriction to subspaces of coloured Minkowski space. These will necessarily break some of the coloured Poincaré symmetries.

The most extreme subspace to be considered is ordinary Minkowski space obtained by setting $x_{I}^{a}=y_{I}=0$. Inspection of (4.4) shows that this breaks the coloured Poincaré symmetry to the usual Poincaré symmetry.

A more interesting restriction is obtained by only setting $x_{I}^{a}=0$. In this case we still have the ordinary Minkowski coordinates $x^{a}$ and the coloured coordinate $y_{I}$ that we would like to think of as the space of an internal degree of freedom of a particle. Setting $x_{I}^{a}=0$ breaks the coloured Poincaré algebra down to the usual Poincaré symmetry and only coloured transformations generated by $N^{I}$ and $Q^{I}$, where we recall that the $N^{I}$ form an $\mathfrak{s u}(N)$ algebra. With only these generations, the equations (4.4) reduce to

$$
\begin{aligned}
& \delta x^{a}=\varepsilon_{b c}{ }^{a} \omega^{b} x^{c}+\alpha^{a}, \\
& \delta y_{I}=-f^{J K}{ }_{I} \sigma_{J} y_{K}+\beta_{I},
\end{aligned}
$$


so that the two coordinates decouple with $x^{a}$ transforming under $\mathfrak{i s o}(2,1)$ and $y_{I}$ transforming under $\mathfrak{s u}(N) \oplus_{\text {adj }} \mathfrak{s u}(N)$.

Let us also consider what happens for the equations of motion (4.51) under the assumption that $x_{I}^{a}=0$ along with a vanishing of the conjugate momentum $p_{a}^{I}=0$. We keep, however, the Euler-Lagrange equation $(4.51 \mathrm{~h})$ for $k_{a I}$. The equations of motion simplify considerably in this reduction. We begin with $(4.51 \mathrm{~h})$ that becomes simply

$$
p^{a} \pi^{I}=0
$$

and there are two branches of solutions: $(i)$ with $\pi^{I}=0$ and (ii) with $p^{a}=0$. Since we wish to describe a massive particle in ordinary Minkowski space, we focus on case $(i)$ where $p^{a} \neq 0$. Then all the remaining equations imply $\lambda^{I}=0$

$$
\begin{aligned}
\dot{x}^{a} & =-2 e p^{a}, \\
\dot{y}^{I} & =2 k_{a I} p^{a}, \\
p^{a} p_{a}+m^{2} & =0 .
\end{aligned}
$$

We see that the first equation is the usual propagation of a particle in Minkowski space and the last equation puts it on the standard mass-shell. The middle equation determines the propagation of the colour coordinate $y^{I}$ and is a bit reminiscent of the Wong equation $[32,59]$ that was developed to describe the coupling of a coloured particle to a Yang-Mills background. However, the equation contains the Lagrange multiplier $k_{a I}$ that should not be interpreted as the background field but takes the value zero. Therefore the colour component $y^{I}$ propagates freely. This is analogous to the coupling of an ordinary charged particle to an electro-magnetic background: The presence of a non-trivial, constant background requires the generalisation of the Poincaré algebra to the so-called Maxwell algebra where $\left[P_{a}, P_{b}\right]=Z_{a b}$, where the non-vanishing commutator of translations corresponds to the electro-magnetic field [28, 29]. Non-constant backgrounds can be incorporated by further generalising the algebra [30,31]. We will comment more on the possible extension of the coloured Poincaré algebra to have non-trivial commutators of coloured translations in the conclusions.

\section{Free coloured particle in AdS background}

In this section, we study the $\mathrm{AdS}_{3}$ analog of the previous construction of the coloured particle in three-dimensional Minkowski space-time. For that, we begin with the coloured $\mathrm{AdS}_{3}$ isometry algebra,

$$
\mathfrak{s u}(N, N) \oplus \mathfrak{s u}(N, N),
$$

which has been considered in $[13,14]$, see also appendix A. One can view this algebra as the colour extension of $\mathfrak{s o}(2,2) \simeq \mathfrak{s u}(1,1) \oplus \mathfrak{s u}(1,1)$, and the flat space algebra $(2.16)$ is related to the above by a Inönü-Wigner contraction. To identify the corresponding particle action, we follow the method of non-linear realisation (or coadjoint action). We are putting the AdS radius $\ell=1$ in this section. Let us first consider the uncoloured case. 


\subsection{Uncoloured particle in AdS}

One can obtain an action for a particle in $\mathrm{AdS}_{d} \cong \mathrm{SO}(d-1,2) / \mathrm{SO}(d-1,1)$ simply by parametrising an $\mathrm{SO}(d-1,2)$ element as

$$
g=t b h,
$$

with an $\mathrm{SO}(d-1)$ element $h$ and

$$
t=\exp \left(x^{0} P_{0}\right) \exp \left(x^{i} P_{i}\right), \quad b=\exp \left(v^{j} M_{0 j}\right),
$$

and following the method of non-linear realisation, see for example [54-57]. The elements $b$ and $h$ together form an element of the Lorentz group $\mathrm{SO}(d-1,1)$. It leads to the particle action with the coordinate system,

$$
d s^{2}=\cosh ^{2} r\left(-d t^{2}+d r^{2}+\sinh r^{2} d \Omega_{d-2}^{2}\right),
$$

where $r=\sqrt{x^{i} x_{i}}$ and $t=x^{0}$. This metric does describe $\operatorname{AdS}_{d}$ space-time, but the Lorentz symmetry $\mathfrak{s o}(d-1,1)$ is not manifest (or linearly realised). Since the colour extension is based on the extension of the $3 \mathrm{~d}$ Lorentz $\mathfrak{s o}(1,2) \simeq \mathfrak{s u}(1,1)$ to $\mathfrak{s u}(N, N)$, this choice of group element is not convenient and we need to find another parametrisation with manifest Lorentz symmetry.

For Lorentz covariance, we can replace $t=\exp \left(x^{0} P_{0}\right) \exp \left(x^{i} P_{i}\right)$ in (5.2) by $t=$ $\exp \left(x^{a} P_{a}\right)$. Or more generally, we can consider $^{16}$

$$
t=f\left(x^{a} P_{a}\right) .
$$

In order that $t$ becomes a group element, we need to require

$$
f(z) f(-z)=1 .
$$

Any real function $f(z)$ with (5.6) would result in good $\mathrm{AdS}_{d}$ particle actions but with different coordinate systems. For instance, in three dimensions the choice $f(z)=e^{z}$ corresponding to $t=e^{x^{a} P_{a}}$ gives the Lagrangian ${ }^{17}$

$$
L=\dot{x}^{\mu} e_{\mu}^{a} p_{a}+e\left(p^{2}+m^{2}\right),
$$

with the dreibein,

$$
e_{\mu}^{a}=\frac{\sinh x}{x} \delta_{\mu}^{a}+\left(1-\frac{\sinh x}{x}\right) \frac{x^{a} x_{\mu}}{x^{2}} .
$$

Here, we used the notation, $x^{2}=x^{a} x_{a}$ and $x=\sqrt{x^{2}}$. Another convenient choice which we shall adopt for the colour extension is of Cayley transform type $f(z)=\frac{1+2 z}{1-2 z}$, that is

$$
t=\frac{1+2 x \cdot P}{1-2 x \cdot P}
$$

\footnotetext{
${ }^{16}$ Note however that the Lie group element $t$ depends not only on the function $f$ but in general also on the representation of $P_{a}$. Here, we take the defining representation.

${ }^{17}$ A similar construction can be found e.g. in [60] where curvature corrections to flat space-time were studied.
} 
with $x \cdot P=x^{a} P_{a}$. Using identities of Pauli matrices, one can find

$$
t^{-1} \dot{t}=\frac{2}{\left(1-x^{2}\right)^{2}}\left[\left(\left(1+x^{2}\right) \dot{x}^{a}-2 x \cdot \dot{x} x^{a}\right) P_{a}-2 \epsilon_{a b c} x^{a} \dot{x}^{b} M^{c}\right],
$$

and the corresponding particle action,

$$
\begin{aligned}
S & =\int d t\left\langle m \mathcal{P}^{0}, g^{-1} \dot{g}\right\rangle=\int d t\left\langle\operatorname{Adj}_{b}^{*}\left(m \mathcal{P}^{0}\right), t^{-1} \dot{t}\right\rangle \\
& =\int d t\left(\sqrt{p_{i}^{2}+m^{2}} e_{\mu}^{0}(x)+p_{i} e_{\mu}^{i}(x)\right) \dot{x}^{\mu},
\end{aligned}
$$

with the dreibein of the form,

$$
e_{\mu}^{a}=2 \frac{\left(1+x^{2}\right) \delta_{\mu}^{a}-2 x_{\mu} x^{a}}{\left(1-x^{2}\right)^{2}}
$$

Combining with the negative energy sector corresponding to the coadjoint element $-m \mathcal{P}^{0}$, we can rewrite the above action with a Lagrange multiplier $e$ as

$$
S[x, p]=\int d t\left(\dot{x}^{\mu} e_{\mu}^{a}(x) p_{a}+e\left(p^{2}+m^{2}\right)\right),
$$

analogously to the flat space case. The dreibein (5.12) gives the metric,

$$
d s^{2}=4 \frac{\left(1+x^{2}\right)^{2} d x^{2}-4(x \cdot d x)^{2}}{\left(1-x^{2}\right)^{4}}
$$

and one can check that it describes $\mathrm{AdS}_{3}$ : with the redefinition,

$$
y^{\mu}=\frac{2 x^{\mu}}{1+x^{2}},
$$

we can bring it to a more standard AdS metric,

$$
d s^{2}=\frac{d y^{2}}{\left(1-y^{2}\right)^{2}} .
$$

The AdS boundary is located at $y^{2}=1$ or equivalently $x^{2}=1$.

\subsection{Coloured particle in AdS}

Let us colour decorate the action (5.11). For that, we decompose an element of $\operatorname{SU}(N, N) \times$ $\mathrm{SU}(N, N)$ as

$$
g=t l
$$

where $l$ is an element of the diagonal $\mathrm{SU}(N, N)$, that is the colour extension of Lorentz subgroup. The other element $t$ belongs to the off-diagonal part which can be interpreted as the colour extension of translation, and we use the parametrisation analogous to (5.9)

$$
t=\frac{1+\mathbb{X}}{1-\mathbb{X}} .
$$


Here, $\mathcal{X}=x_{\widehat{I}}^{a} P_{a}^{\widehat{I}}+y_{I} Q^{I}$ is an $2 N \times 2 N$ matrix with the condition (2.18). In principle, we can take any function $f(\mathbb{X})$ with (5.6), but the expression of $\dot{h}$ becomes complicated or implicit for a generic $f(z)$. Different choices of $f(\mathbb{X})$ corresponds to a field redefinition of $\mathbb{X}$, so we consider only the simple case above.

Choosing the same representative of the coadjoint orbit, $m \mathcal{P}_{0}^{0}$, as in flat space case, we find

$$
S=m \int d t\left\langle\operatorname{Ad}_{l}^{*}\left(m \mathcal{P}_{0}^{0}\right), t^{-1} \dot{t}\right\rangle,
$$

as the action for coloured particle in $\mathrm{AdS}_{3}$. Using the isomorphism between $\mathfrak{s u}(N, N)$ and $\mathfrak{s u}(N, N)^{*}$, we can rewrite the above as

$$
S=\frac{1}{2 N} \int d t \operatorname{Tr}\left[l \widehat{\mathbb{P}} l^{-1} t^{-1} \dot{t}\right]
$$

where each element is expressed in the fundamental representation of $\mathrm{SU}(N, N)$ and $\widehat{\mathbb{P}}$ is as given in (4.52). The $2 N \times 2 N$ matrix

$$
\mathbb{P}=l \widehat{\mathbb{P}} l^{-1},
$$

satisfies the condition $\mathbb{P}^{2}+m^{2} \rrbracket=0$, but the latter contains other orbits besides the one determined by $m \mathcal{P}_{0}^{0}$. The issue of algebraically describing the orbit at the cost of including other ones is the same as in the flat space case. In the end, we find the $\mathrm{AdS}_{3}$ analog of the coloured action (4.26) as

$$
S=\frac{1}{N} \int d t \operatorname{Tr}\left[\mathbb{P}\left(\frac{1}{1+\mathbb{X}} \dot{\mathbf{X}} \frac{1}{1-\mathbb{X}}\right)-\mathbb{L}\left(\mathbb{P}^{2}+m^{2} \mathbb{q}\right)\right],
$$

where $\mathbb{X}, \mathbb{P} \in \mathfrak{s u}(N, N)$ and $\mathbb{L} \in \mathfrak{u}(N, N)$. By a simple field redefinition, the action above can be written as

$$
S=\frac{1}{N} \int d t \operatorname{Tr}\left[\mathbb{P} \dot{\mathcal{X}}-\mathbb{L}\left([(1-\mathbb{X}) \mathbb{P}(1+\mathbb{X})]^{2}+m^{2} \mathbb{a}\right)\right],
$$

employing the standard Liouville form.

\section{Conclusions}

In this paper, we have defined a coloured extension of the three-dimensional Poincaré algebra. This algebra, after removing its center, is isomorphic to $\mathfrak{s u}(N, N) €_{\text {adj }} \mathfrak{s u}(N, N)$, where the first $\mathfrak{s u}(N, N)$ is the colouring of the Lorentz symmetry that acts on the coloured translations in the adjoint representation.

We have defined Poincaré gravity based on this algebra using a Chern-Simons formulation where an interesting interplay between the colour degrees of freedom and the ordinary gravity degrees of freedom is implied by the structure of the algebra (and choice of bilinear form). ${ }^{18}$

The coloured Poincaré algebra can also be used to define coloured massive and massless free particles. There are different types of coloured particles depending on the choice of

\footnotetext{
${ }^{18}$ For the coloured $\mathrm{AdS}_{3}$ case, see $[13,14]$.
} 
co-adjoint orbit of the coloured Poincaré algebra, generalising positive and negative energy particles. There are various different Lagrangians depending on how many orbits one wishes to describe at the same time. We have also considered the extension to particles in coloured AdS space based on the algebra originally constructed in [13].

There are several interesting avenues to explore in order to further investigate the structures introduced here. One question, raised in the introduction, is that of relating our analysis to the classical description of coloured particles in terms of the Wong equation [32, $59]$ and we have taken some first steps in this direction in section 4.8 , where we considered subspaces of coloured Minkowski space. Even more interesting would be to consider a further extension of the coloured Poincaré algebra to a coloured Maxwell algebra. The uncoloured Maxwell algebra allows for the description of a charged particle in an electromagnetic background [28-31] and we expect the coloured Maxwell algebra to be the right framework for (constant) Yang-Mills backgrounds.

The coloured Maxwell algebra has further generators $Z^{\widehat{K}}, Z_{a}^{\widehat{K}}$ on top of those of the Poincaré algebra. The commutation relations of the coloured Maxwell algebra can be obtained via semi-group expansion (see footnote 6 ) of the algebra $\mathfrak{u}(N, N)$ with semi-group $S_{E}^{(2)}=\left\{\lambda_{0}, \lambda_{1}, \lambda_{2}, \lambda_{3}\right\}$

$$
\begin{aligned}
& {\left[M_{a}^{\widehat{I}}, M_{b}^{\widehat{J}}\right]=\frac{1}{2} \varepsilon_{a b}{ }^{c} \widehat{d}^{\widehat{I J}} \widehat{K}_{c}^{\widehat{K}}-\frac{1}{4} \eta_{a b} \widehat{f}^{\widehat{I} \widehat{K}}{ }_{\widehat{K}} N^{\widehat{K}},} \\
& {\left[M_{a}^{\widehat{I}}, N^{\widehat{J}}\right]=\widehat{f}_{\widehat{I} \widehat{J}}^{\widehat{K}} M_{a}^{\widehat{K}}, \quad\left[N^{\widehat{I}}, N^{\widehat{J}}\right]=\widehat{f}{ }_{\widehat{K}}^{\widehat{I}} N^{\widehat{K}},} \\
& {\left[M_{a}^{\widehat{I}}, P_{b}^{\widehat{J}}\right]=\frac{1}{2} \varepsilon_{a b}{ }^{c} \widehat{d}^{\widehat{I J}}{ }_{\widehat{K}} P_{c}^{\widehat{K}}-\frac{1}{4} \eta_{a b} \widehat{f}^{\widehat{I J}}{ }_{\widehat{K}} Q^{\widehat{K}},} \\
& {\left[M_{a}^{\widehat{I}}, Q^{\widehat{J}}\right]=\left[N^{\widehat{I}}, P_{a}^{\widehat{J}}\right]=\widehat{f}^{\widehat{I} \widehat{J}} \widehat{K} P_{a}^{\widehat{K}},} \\
& {\left[N^{\widehat{I}}, Q^{\widehat{J}}\right]=\widehat{f}_{\widehat{K}}^{\widehat{I} \widehat{K}} Q^{\widehat{K}},} \\
& {\left[P_{a}^{\widehat{I}}, P_{b}^{\widehat{J}}\right]=\left[M_{a}^{\widehat{I}}, Z_{b}^{\widehat{J}}\right]=\frac{1}{2} \varepsilon_{a b}{ }^{c} \widehat{d}^{\widehat{I J}} \widehat{K} Z_{c}^{\widehat{K}}-\frac{1}{4} \eta_{a b} \widehat{f}^{\widehat{I J}}{ }_{\widehat{K}} Z^{\widehat{K}},} \\
& {\left[P_{a}^{\widehat{I}}, Q^{\widehat{J}}\right]=\left[M_{a}^{\widehat{I}}, Z^{\widehat{J}}\right]=\left[N^{\widehat{I}}, Z_{a}^{\widehat{J}}\right]=\widehat{f}^{\widehat{I} \widehat{J}} \widehat{K} Z_{a}^{\widehat{K}},} \\
& {\left[Q^{\widehat{I}}, Q^{\widehat{J}}\right]=\left[N^{\widehat{I}}, Z^{\widehat{J}}\right]=\widehat{f}_{\widehat{I}}^{\widehat{I}} Z^{\widehat{K}} .}
\end{aligned}
$$

All generators here are anti-hermitian and the generators of the semi-group $\lambda_{i}$ are hermitian. This algebra can also be embedded in a free Lie algebra construction of the type considered in [31]. The algebra (6.1) is a quotient of the semi-direct sum of the $\mathfrak{u}(N, N)$ with the free Lie algebra generated by the translations $\left(P_{a}^{\widehat{I}}, Q^{\widehat{I}}\right)$ that transform in the adjoint of $\mathfrak{u}(N, N)$. One could similarly consider other quotients leading to generalisations of the $\mathcal{B}_{\infty}$ algebra studied in [61, 62] or to half a Kac-Moody algebra [62]. 
Besides describing particles one may wonder whether there are coloured extensions of the actions of extended objects such as strings or branes (see, e.g., [57]). In the nonrelativistic limit one might also have to change the algebra, see for example [63].

Our analysis was restricted to classical particle dynamics and it would be interesting to extend the models to (free or interacting) field theories. A first question is which of our particle actions to quantise. As the different actions in section 4 comprise different orbits, this will correspond to including different irreducible representations at the quantum level. ${ }^{19}$ The constraints (4.23) and (4.27) are quadratic in the canonical variables and will lead to quadratic wave equations on the space of wavefunctions $\Psi(\mathbb{P})$. However, as is evident from the detailed analysis of the case with $\mathrm{SU}(2)$ colour in section 4.6, the wave equation on the reduced phase space can be of higher order. After solving most of the matrix-valued constraints, we arrived at the final constraint (4.79) arising in the case $N=2$ for the massive particle. This scalar equation can be read as a quartic condition on the momenta and therefore should translate into a fourth order Klein-Gordon equation in canonical quantisation of this mass-shell constraint. We note the general constraint (4.27) is matrixvalued and therefore would correspond to a matrix-valued Klein-Gordon operator whose direct interpretation is as an orbit condition. This situation is similar to what happens in $\operatorname{Sp}(2 N)$-invariant (higher-spin) field theory [65] (see [66] for a review), where the matrixvalued field-equation can be interpreted as the condition of the minimal nilpotent orbit of $\operatorname{Sp}(2 N)$ (see [67] for discussions about the minimal nilpotent orbits of classical Lie algebras in the context of higher spin field theory).

If the coloured particle is properly quantised, it will correspond to a representation of the group $\mathrm{cPoin}_{3}(N)$. This representation can be constructed analogously to the induced scalar representation of the ordinary Poincaré algebra: the representation space is the space of square integrable functions on the orbit with a proper $\mathrm{SU}(N, N)$ invariant measure on it. The invariant measure can be obtained by solving the matrix-valued "momentum-shell constraint" $\mathbb{P}^{2}+m^{2} \rrbracket=0$, and hence the hermitian product can be defined as

$$
\left\langle\psi_{1}, \psi_{2}\right\rangle=\int d \mathbb{P} \delta\left(\mathbb{P}^{2}+m^{2} \square\right) \psi_{1}(\mathbb{P})^{*} \psi_{2}(\mathbb{P}),
$$

where $d \mathbb{P}$ is the $\left((2 N)^{2}-1\right)$-dimensional measure on $\mathfrak{s u}(N, N)$, and $\delta\left(\mathbb{P}^{2}+m^{2} \square\right)$ is the $\left(2 N^{2}-1\right)$-dimensional delta distribution supported on the momentum orbits that we have described above. The action of an element of the coloured Poincaré group, $(\mathbb{A}, \mathbb{A}) \in$ $\mathrm{SU}(N, N) \ltimes \mathrm{SU}(N, N)$, is given by

$$
(\mathrm{U}(\Lambda, \mathbb{A}) \psi)(\mathbb{P})=e^{i \operatorname{tr}\left(\mathbb{A} \Lambda^{-1} \mathbb{P} \wedge\right)} \psi\left(\bigwedge^{-1} \mathbb{P} \wedge\right)
$$

\footnotetext{
${ }^{19}$ When an action contains several orbits the classical phase space is in general a Poisson rather than a symplectic manifold and it would be interesting to apply Kontsevich's deformation quantisation methods [64] in this context.
} 
and the unitarity follows from the invariance of the measure and the delta distribution,

$$
d\left(\bigwedge^{-1} \mathbb{P} \wedge\right)=d \mathbb{P}, \quad \delta\left(\bigwedge^{-1}\left(\mathbb{P}^{2}+m^{2} \llbracket\right) \wedge\right)=\delta\left(\mathbb{P}^{2}+m^{2} \llbracket\right) .
$$

A similar construction can be done in the $\mathrm{AdS}_{3}$ case. ${ }^{20}$

We also note that we are here dealing with an extension of a space-time symmetry and may wonder how this is compatible with the Coleman-Mandula theorem. Although we have not explicitly constructed a field theory with coloured Poincaré invariance, we do not expect any contradiction to the theorem since the space-time has been vastly extended from Minkowski space-time to coloured Minkowski space. We consider this extension to be a bit similar in spirit to what happens in supersymmetry (or higher spin theory).

Finally, our construction was strongly based on three-dimensional space-time and one big challenge is to generalise the construction to arbitrary space-time dimensions. As discussed here and in [13, 14], non-abelian colour decorations require associative extensions of the algebra of isometries to start with. Such extensions usually involve generators in non-trivial representations of the isometry algebra (see, e.g., [69]). Exceptions, i.e. extensions of isometry algebras involving only generators in trivial representations (that is, corresponding to symmetries of vector gauge fields), are possible in two dimensions (as discussed in section 2) and three dimensions (as we studied in this paper) as well as in five dimensions: the $\mathrm{AdS}_{5}$ isometry algebra $\mathfrak{s o}(2,4) \simeq \mathfrak{s u}(2,2)$ (which is the same as conformal algebra in four dimensions) can be extended by a single generator to $\mathfrak{u}(2,2)$ allowing for colour generalisation to $\mathfrak{u}(2 M, 2 M) \cong \mathfrak{u}(2,2) \otimes \mathfrak{u}(M)$. Interpreting the latter as the coloured $\mathrm{AdS}_{5}$ isometry algebra, the momentum orbits that we have considered in this paper can be viewed as the phase spaces of coloured particles in $\mathrm{AdS}_{5}$. It is intriguing to realise that the $N=2$ coloured $\mathrm{AdS}_{3}$ particle, having the phase space $\mathcal{O}_{ \pm 2} \times \mathcal{O}_{ \pm 2}$, can be interpreted as a particle on two copies of $\mathrm{AdS}_{5}$ since the phase of the latter is simply one factor $\mathcal{O}_{ \pm 2}$. Here, $\mathcal{O}_{ \pm 2}$ refer to the (momentum) orbits discussed around (4.81).

\footnotetext{
${ }^{20}$ More specifically, the colour symmetry algebra for $\operatorname{AdS}_{3}$ is $\mathfrak{s u}(N, N) \oplus \mathfrak{s u}(N, N)$, and the unitary representation would be of the form $\pi \otimes \pi$ where $\pi$ is a unitary irreducible representation of $\mathfrak{s u}(N, N)$ (we take the same representation for both sides of $\mathfrak{s u}(N, N)$ because we are describing scalar particles). To identify $\pi$, we can rely on the GK dimension, which is the same in both flat and $\mathrm{AdS}_{3}$ space. Since GK dimension is additive under tensor product, the GK dimension of $\pi$ should be $N^{2}$, i.e., half of the dimension $2 N^{2}$ of the momentum orbit. In the massless case, we have seen that the constraint $\mathbb{P}^{2}=0$ contains momentum orbits of dimensions $4 N k-2 k^{2}$ with $k=1, \ldots, N$ (except for the trivial orbit $k=0$ ). Therefore, the representations $\pi_{k}$ corresponding to massless coloured particle in $\mathrm{AdS}_{3}$ should have GK dimension $2 N k-k^{2}$. Interestingly, such representations can be realised by using the reductive dual pair $(\mathrm{U}(N, N), \mathrm{U}(k))$, where we take the trivial representation for the $\mathrm{U}(k)$. For a review of dual pairs see [68]. Disregarding the diagonal $\mathrm{U}(1)$ of $\mathrm{U}(N, N)$, the corresponding $\mathrm{SU}(N, N)$ representation is the generalised Verma module induced from the one dimensional representation of the maximal compact subgroup $\mathrm{U}(1) \times \mathrm{SU}(N) \times \mathrm{SU}(N) \subset \mathrm{SU}(N, N)$ with the $\mathfrak{u}(1)$ eigenvalue $k N$. Turning back to the massive case, the representation is again given by the dual pair $(\mathrm{U}(N, N), \mathrm{U}(N))$ where we take other one-dimensional representations with eigenvalue $n$ for $\mathrm{U}(1) \subset \mathrm{U}(N)$ while keeping the trivial representation for $\mathrm{SU}(N) \subset \mathrm{U}(N)$. Then, the corresponding $\mathrm{SU}(N, N)$ representation is the generalised Verma module induced from the one dimensional representation of $\mathrm{U}(1) \times \mathrm{SU}(N) \times \mathrm{SU}(N) \subset \mathrm{SU}(N, N)$ with the $\mathfrak{u}(1)$ eigenvalue $n+N^{2}$. In the flat limit where the AdS radius $\ell$ tends to $\infty$, we take the limit $n \rightarrow \infty$ while keeping $n / \ell$ fixed as a constant proportional to the mass $m$.
} 
We also note that our analysis was restricted to scalar particles and it would be interesting to consider particles with spin as well. Spin corresponds to a non-trivial representation of the stability group which is here also enlarged compared to the uncoloured case. For instance, in the case of the representative momentum (4.52), the stability algebra was $\mathfrak{u}(1) \oplus \mathfrak{s u}(N) \oplus \mathfrak{s u}(N)$ and taking a non-trivial representation of $\mathfrak{u}(1)$ would be analogous to ordinary spin but one could also envisage more colourful versions of spin by taking non-trivial representations of the $\mathfrak{s u}(N)$ parts. Extensions to superalgebras could also be interesting to explore.

\section{Acknowledgments}

We would like to thank Thomas Basile for useful discussions. JG acknowledges the hospitality at Max-Planck-Institut für Gravitationsphysik (Albert-Einstein-Institut), Potsdam where this work was started. EJ and KM are grateful to the Erwin Schrödinger International Institute for Mathematics and Physics for the hospitality during the program on "Higher Spins and Holography". The work of JG has been supported in part by MINECO FPA2016-76005-C2-1-P and PID2019-105614GB-C21 and from the State Agency for Research of the Spanish Ministry of Science and Innovation through the Unit of Excellence Maria de Maeztu 2020-203 award to the Institute of Cosmos Sciences (CEX2019-000918M). The work of EJ was supported by National Research Foundation (Korea) through the grant NRF-2019R1F1A1044065. KM is supported by the European Union's Horizon 2020 research and innovation programme under the Marie Skłodowska-Curie grant number 844265. Part of this work was carried out while KM was at Max-Planck Institut für Gravitationsphysik and Scuola Normale Superiore di Pisa.

\section{A Derivation from embedding in AdS algebra}

The uplift of the Poincaré algebra to an associative algebra proceeds in two steps. First we embed the (2+1)-dimensional Poincaré algebra in the corresponding AdS algebra $\mathfrak{s o}(2,2) \cong$ $\mathfrak{s l}(2, \mathbb{R}) \oplus \mathfrak{s l}(2, \mathbb{R})$ that we then extend to the associative algebra $\mathfrak{a}=\mathfrak{u}(1,1) \oplus \mathfrak{u}(1,1)$. The coloured Poincaré algebra in $D=3$ is then obtained in the contracting limit where the AdS radius is sent to infinity.

To see this in more detail consider first the AdS algebra

$$
\left[M_{a}, M_{b}\right]=\varepsilon_{a b}^{c} M_{c}, \quad\left[M_{a}, P_{b}\right]=\varepsilon_{a b}^{c} P_{c}, \quad\left[P_{a}, P_{b}\right]=\frac{1}{\ell^{2}} \varepsilon_{a b}{ }^{c} M_{c},
$$

where $M_{a}=\frac{1}{2} \varepsilon_{a b c} M^{b c}$ are the dual Lorentz generators, $P_{a}$ the translation generators and $\ell$ denotes the AdS radius. Indices are raised and lowered with the $(-++)$ Minkowski metric in $D=3$. To bring out the $\mathfrak{s l}(2, \mathbb{R}) \oplus \mathfrak{s l}(2, \mathbb{R})$ form of this algebra one defines the standard combinations

$$
J_{a}=\frac{1}{2}\left(M_{a}+\ell P_{a}\right), \quad \tilde{J}_{a}=\frac{1}{2}\left(M_{a}-\ell P_{a}\right),
$$


such that

$$
\left[J^{a}, J^{b}\right]=\varepsilon_{a b}^{c} J_{c}, \quad\left[\tilde{J}^{a}, \tilde{J}^{b}\right]=\varepsilon_{a b}{ }^{c} \tilde{J}_{c}, \quad\left[J^{a}, \tilde{J}^{b}\right]=0,
$$

making the two commuting $\mathfrak{s l}(2, \mathbb{R})$ manifest.

The AdS algebra (A.1) can also be written via a tensor product of $\mathfrak{s l}(2, \mathbb{R})$ with an associative abelian algebra $\mathfrak{a}_{\ell}$ on two generators $\mathcal{I}$ and $\mathcal{J}$ satisfying

$$
\mathcal{I}^{2}=\mathcal{I}, \quad \mathcal{J}^{2}=\frac{1}{\ell^{2}} \mathcal{I}, \quad \mathcal{I} \mathcal{J}=\mathcal{J} \mathcal{I}=\mathcal{J}
$$

Since $\mathfrak{a}_{\ell}$ is abelian, one can turn $\mathfrak{s l}(2, \mathbb{R}) \otimes \mathfrak{a}_{\ell}$ into a Lie algebra and this Lie algebra is isomorphic to the AdS algebra. ${ }^{21}$ As the final step one defines

$$
\mathfrak{A}_{\ell}=\mathfrak{u}(1,1) \otimes \mathfrak{a}_{\ell}
$$

as an associative algebra. Sending $\ell \rightarrow \infty$ for the flat space limit and tensoring with the associative algebra $\mathfrak{u}(N)$ one obtains the coloured Poincaré algebra in $D=3$ dimensions shown in (2.13).

\section{B Free massive Poincaré particle}

Here, we give a short exposition of the Lagrangian of a free massive particle built on the standard Poincaré group using non-linear realisations, see e.g. [57]. For easier comparison with the body of the paper we do everything in $D=3$ space-time dimensions using the algebra $(2.5)$ and write the algebra $\mathfrak{s o}(2,1) \cong \mathfrak{s u}(1,1)$ as $(2 \times 2)$-matrices.

Massive particle. We begin with the construction from non-linear realisations. For a massive particle in rest frame, the momentum eigenvalues take the form $p_{a}=(m, 0,0)$ with $\mathrm{SO}(2) \cong \mathrm{U}(1)$ stabiliser in the Lorentz group generated by $M_{0}$. The local subgroup $H$ of the non-linear realisation is thus $\mathrm{SO}(2)$ and we write group elements in the form

$$
g=e^{x^{a} P_{a}} b h,
$$

with $b=e^{v^{i} M_{i}}(i=1,2)$ the boost by the broken generators in the Lorentz group. We choose the gauge $h=\rrbracket$ for the $G / H$ coset representative.

The Poincaré algebra valued Maurer-Cartan form for this gauge-fixed coset element is

$$
\Omega=g^{-1} d g=b^{-1}\left(d x^{a} P_{a}\right) b+b^{-1} d b=\Omega_{(P)}^{a} P_{a}+\Omega_{(M)}^{a} M_{a} .
$$

The components of the Maurer-Cartan form are by construction invariant under the global Poincaré group acting from the left on the group element (B.1).

If one wants to construct a Lagrangian that is also invariant under the right-action of the unbroken local $\mathrm{SO}(2)$ subgroup of the Lorentz group, one can therefore choose any component of the Maurer-Cartan form that is invariant under $M_{0}$. In the present case, there are two such candidates: $\Omega_{(P)}^{0}$ and $\Omega_{(M)}^{0}$. The former is properly invariant while the latter transforms as a gauge field into a total derivative and thus is only quasi-invariant. This quasi-invariance is special to $D=3$ dimensions, in general dimensions there is no such singlet from the Lorentz algebra decomposed under spatial rotations. ${ }^{22}$ We shall therefore

\footnotetext{
${ }^{21}$ For $\ell=1$, the algebra (A.4) is identical to the semi-group $S_{M}^{(1)}$ studied in [41].

${ }^{22}$ The form $\Omega_{(M)}^{0}$ is a Wess-Zumino term in three space-time dimensions [70-73].
} 
ignore $\Omega_{(M)}^{0}$ and take the Lagrangian to be given by the pull-back of the component $\Omega_{(P)}^{0}$ to the world-line of the particle: ${ }^{23}$

$$
L d t=m\left[\Omega_{(P)}^{0}\right]^{*} .
$$

We note that the component $\Omega_{(P)}^{0}$ can also be obtained by pairing the space of translations, generated by the $P_{a}$, with its dual. In other words, using as the dual basis $\mathcal{P}^{a}$ with pairing

$$
\left\langle P_{a}, \mathcal{P}^{b}\right\rangle=\delta_{a}^{b}
$$

one has

$$
\Omega_{(P)}^{0}=\left\langle\Omega_{(P)}, \mathcal{P}^{0}\right\rangle=\left\langle b^{-1}\left(d x^{a} P_{a}\right) b, \mathcal{P}^{0}\right\rangle=\left\langle\operatorname{Ad}_{b^{-1}}\left(d x^{a} P_{a}\right), \mathcal{P}^{0}\right\rangle=\left\langle\left(d x^{a} P_{a}\right), \operatorname{Ad}_{b^{-1}}^{*} \mathcal{P}^{0}\right\rangle
$$

where we have used $\operatorname{Ad}_{b^{-1}}$ to denote the 'adjoint' action of the Lorentz group element $b^{-1}$ on its vector representation when viewing the Poincaré group as a semi-direct product. Similarly, $\mathrm{Ad}_{b^{-1}}^{*}$ is the co-adjoint action on the dual space.

Writing $x^{a} P_{a}=\mathbb{X}$ we therefore have the equivalent form of the Lagrangian (B.3)

$$
L=\langle\mathbb{P}, \dot{\mathcal{K}}\rangle,
$$

where $\mathbb{P}$ is any element in the Lorentz group orbit of $m \mathcal{P}^{0}$. Here, it is important that the orbit of $\mathcal{P}^{0}$ can be written as the group coset $\mathrm{SO}(2,1) / \mathrm{SO}(2)$ by the orbit stabiliser theorem. This orbit can be parametrised by some parameters $v^{i}$ that then appear algebraically in the Lagrangian: the boost parameters. The derivative in $\dot{\mathcal{K}}$ denotes the derivative with respect to the parameter of the world-line and so we have explicitly carried out the pull-back.

Other orbits of the action of the Lorentz group on the space of momenta can also be considered and the form (B.6) is universal in all cases. If the orbit is of the form $\mathrm{SO}(2,1) / H$ for some subgroup $H$ then the Lagrangian corresponds to the non-linear realisation of the Poincaré group with local subgroup $H$.

This can also be treated as the $N=1$ case of the matrix analysis in the main body of the paper. We take as reference momentum

$$
\widehat{\mathbb{P}}=m \mathcal{P}^{0}=m\left(\begin{array}{cc}
\mathrm{i} & 0 \\
0 & -\mathrm{i}
\end{array}\right) .
$$

The generalised boost (4.33b) becomes

$$
b=\exp \left[\frac{1}{2}\left(\begin{array}{ll}
0 & v \\
\bar{v} & 0
\end{array}\right)\right]=\left(\begin{array}{l}
\cosh \frac{|v|}{2} v \frac{\sinh \frac{|v|}{2}}{|v|} \\
\bar{v} \frac{\sinh \frac{|v|}{2}}{|v|} \cosh \frac{|v|}{2}
\end{array}\right)
$$

\footnotetext{
${ }^{23}$ Note that $\Omega_{(P)}^{0}$ is nothing but the time column of a Lorentz boost acting on a general momentum.
} 
with $v \in \mathbb{C}$. The orbit of the reference momentum is

$$
b \widehat{\mathbb{P}} b^{-1}=m \mathrm{i}\left(\begin{array}{c}
\cosh |v|-v \frac{\sinh |v|}{|v|} \\
\bar{v} \frac{\sinh |v|}{|v|}-\cosh |v|
\end{array}\right)=\left(\begin{array}{cc}
\mathrm{i} \sqrt{|p|^{2}+m^{2}} & p \\
\bar{p} & -\mathrm{i} \sqrt{|p|^{2}+m^{2}}
\end{array}\right),
$$

where we have introduced $p=-m \mathrm{i} \frac{\sinh |v|}{|v|} v$ as a change of coordinates for the algebraically appearing boost parameters.

The velocity vector is the matrix

$$
\dot{\boldsymbol{k}}=\left(\begin{array}{cc}
\mathrm{i} \dot{x}^{0} & \dot{z} \\
\dot{\bar{z}} & -\mathrm{i} \dot{x}^{0}
\end{array}\right)
$$

where $z=x^{1}+\mathrm{i} x^{2}$. With this the Lagrangian

$$
\begin{aligned}
L & =\frac{1}{2} \operatorname{Tr}\left(\dot{\mathcal{X}} b \widehat{\mathbb{P}} b^{-1}\right)=m\left[\dot{x}^{0} \cosh |v|+\operatorname{Im}(\dot{z} \bar{v}) \frac{\sinh |v|}{|v|}\right] \\
& =-\dot{x}^{0} \sqrt{|p|^{2}+m^{2}}+\operatorname{Re}(\dot{z} \bar{p}) .
\end{aligned}
$$

The equation of motion obtained by varying with respect to the algebraic variable $p$ is

$$
\frac{\dot{x}^{0}}{\sqrt{|p|^{2}+m^{2}}} \bar{p}=\dot{\bar{z}}
$$

Solving this for $p$ and substituting back into the Lagrangian leads to the expected

$$
L=-m \sqrt{\left(\dot{x}^{0}\right)^{2}-|\dot{z}|^{2}} .
$$

Going back to the action (B.11) we can also perform another analysis that is closer to the main body of the text. For this we vary the Lagrangian (B.11) with respect to $x^{0}$. This leads to

$$
\sqrt{|p|^{2}+m^{2}}=p^{0}
$$

where $p^{0}$ is a constant of integration while the spatial $p$ is still a dynamical variable. As $p^{0}$ sits at the right place in the momentum matrix we deduce that it transforms as the zero component of the contravariant momentum $p^{a}$ would. Enforcing the square of the above relation with a Lagrange multiplier $e$ leads to the Lagrangian

$$
L=\dot{x}^{a} p_{a}+e\left(p^{a} p_{a}+m^{2}\right) .
$$

This Lagrangian has manifest Poincaré invariance. In this Lagrangian, all components of $p_{a}$ are algebraic variables and we can integrate them out to obtain

$$
L=-\frac{1}{4 e} \dot{x}^{a} \dot{x}_{a}+e m^{2}=-m \sqrt{-\dot{x}^{a} \dot{x}_{a}},
$$

where in the second step we have also integrated out $e$ (with a choice of square root). This Lagrangian agrees with (B.13) above. 
Massless case. Let us also consider the case of a massless particle in this formulation. The representative momentum is now given by

$$
\widehat{\mathbb{P}}=\frac{1}{2}\left(\begin{array}{cc}
\mathrm{i} E & E \\
E & -\mathrm{i} E
\end{array}\right), \quad \operatorname{tr}(\widehat{\mathbb{P}} \widehat{\mathbb{P}})=0
$$

and it is stabilised by the one-parameter group generated by $M_{0}+M_{1}=\left(\begin{array}{cc}\mathrm{i} & 1 \\ 1 & -\mathrm{i}\end{array}\right)$.

The boost can still be chosen as (B.8) and an arbitrary element of the orbit of the reference momentum is

$$
b \widehat{\mathbb{P}} b^{-1}=\left(\begin{array}{cr}
i \omega & e^{\mathrm{i} \theta} \omega \\
e^{-\mathrm{i} \theta} \omega & -\mathrm{i} \omega
\end{array}\right)
$$

in a convenient parametrisation. The Lagrangian becomes

$$
L=\frac{1}{2} \operatorname{Tr}\left(\dot{\mathrm{X}} b \widehat{\mathbb{P}} b^{-1}\right)=-\omega\left(\dot{x}^{0}-\operatorname{Re}\left(e^{-i \theta} \bar{z}\right)\right)
$$

The equation of motion when varying $\theta$ is

$$
e^{2 \mathrm{i} \theta}=\frac{\dot{z}}{\dot{\bar{z}}}
$$

leading to

$$
L=-\omega\left(\dot{x}^{0}-\sqrt{|\dot{z}|^{2}}\right) .
$$

This enforces correctly the constraint $\dot{x}^{a} \eta_{a b} \dot{x}^{b}=0$, but is not quite the usual form for a massless Poincaré particle.

Let us also go the alternative route for the Lagrangian (B.19), meaning that we vary with respect to $x^{0}$ first. This forces the energy of the particle to be constant

$$
\omega=p^{0}
$$

for some constant $p^{0}$. We enforce the square of this relation as a constraint

$$
L=p_{0} \dot{x}^{0}+p_{i} \dot{x}^{i}+e\left(\omega^{2}-p_{0}^{2}\right)=p_{a} \dot{x}^{a}+e p_{a} p^{a},
$$

where we have used that $\omega e^{\mathrm{i} \theta}=p^{1}+\mathrm{i} p^{2}$ represents the spatial momentum. Integrating out the covariant $p^{a}$ leads to expected action

$$
L=-\frac{1}{4 e} \dot{x}^{a} \dot{x}_{a} .
$$

Both Lagrangians (B.21) and (B.24) can be obtained from the original (B.19) (modulo the fact that for (B.24) a square for anti-particles was introduced). Let us study their equivalence in general. The equations of motion implied by (B.21) are

$$
\begin{aligned}
\dot{x}^{0}-|\dot{\mathbf{x}}| & =0, \\
\frac{d}{d \tau} \omega & =0, \\
\frac{d}{d \tau}\left(\frac{\dot{\mathbf{x}}}{|\dot{\mathbf{x}}|}\right) & =0,
\end{aligned}
$$


while from (B.24) we get

$$
\begin{aligned}
\dot{x}^{a} \dot{x}_{a} & =0, \\
\frac{d}{d \tau}\left(\frac{\dot{x}^{a}}{e}\right) & =0 .
\end{aligned}
$$

We first observe that (B.25a) and (B.26a) are equivalent, up to the choice of square root. Let us set

$$
\omega=\frac{\dot{x}^{0}}{e} \quad \Leftrightarrow \quad e=\frac{\dot{x}^{0}}{\omega} \underset{(\mathrm{B} .25 \mathrm{a})}{=} \frac{|\dot{\mathbf{x}}|}{\omega}
$$

and this is conserved consistently by (B.25b) and the first component of (B.26b). The spatial components of (B.26b) are

$$
\frac{d}{d \tau}\left(\frac{\dot{\mathbf{x}}}{e}\right)=\frac{d}{d \tau}\left(\omega \frac{\dot{\mathbf{x}}}{|\dot{\mathbf{x}}|}\right)=\omega \frac{d}{d \tau}\left(\frac{\dot{\mathbf{x}}}{|\dot{\mathbf{x}}|}\right)=0
$$

consistently with (B.25c) when using (B.25b).

\section{General remarks on particle actions}

In this appendix, we collect some general remarks on the different ways of writing particle actions based on symmetries.

We go back to the action of nonlinear realisation or the coadjoint orbit,

$$
S=\int d t\left\langle\varphi, g^{-1} \frac{d}{d t} g\right\rangle
$$

where $g \in G$ and $\varphi \in \mathfrak{g}^{*}$. The coadjoint action of $a \in G$ is defined as

$$
\left\langle\operatorname{Ad}_{a}^{*} \varphi, T\right\rangle=\left\langle\varphi, \operatorname{Ad}_{a^{-1}} T\right\rangle, \quad \forall T \in \mathfrak{g} .
$$

For a given $\varphi$, let us denote the stabiliser subgroup by $G_{\varphi}=\left\{a \in G \mid \operatorname{Ad}_{a}^{*} \varphi=\varphi\right\}$ and its Lie algebra by $\mathfrak{g}_{\varphi}=\left\{T \in \mathfrak{g} \mid \operatorname{Ad}_{T}^{*} \varphi=0\right\}$. The dimension of the coadjoint orbit is simply,

$$
\operatorname{dim} \mathcal{O}_{\varphi}=\operatorname{dim} \mathfrak{g} / \mathfrak{g}_{\varphi}=\operatorname{dim} \mathfrak{g}-\operatorname{dim} \mathfrak{g}_{\varphi} .
$$

As an example, consider $G$ as the $D$-dimensional Poincaré group $\operatorname{ISO}(D-1,1)$ and $\varphi=$ $m \mathcal{P}^{0}$ where $\mathcal{P}^{0}$ is the dual basis generator corresponding to $P_{0}$. Then, ${ }^{24}$

$$
\mathfrak{p o i n}_{m \mathcal{P}^{0}}=\operatorname{Span}\left\{M_{i j}, P_{0}\right\}, \quad \mathfrak{p o i n} / \mathfrak{p o i n}{ }_{m P_{0}^{*}} \sim \operatorname{Span}\left\{M_{0 i}, P_{i}\right\} .
$$

Hence, we find the orbit has $2 \times(D-1)$ dimensions, i.e. it is a phase space for $(D-1)$ dimensional mechanical system so can describe the motion of a massive particle in $D$ dimensional space-time. If we want to see this more explicitly, we can consider an element of $g \in G$ such that

$$
g=a b, \quad b \in G_{\varphi},
$$

\footnotetext{
${ }^{24}$ Note that this contrasts with$$
\operatorname{poin}_{P_{0}}=\operatorname{Span}\left\{M_{i j}, P_{0}, P_{i}\right\}, \quad \text { poin } / \text { poin }_{P_{0}} \sim \operatorname{Span}\left\{M_{0 i}\right\} .
$$ 
then,

$$
\left\langle\varphi, g^{-1} d g\right\rangle=\left\langle\varphi, a^{-1} d a\right\rangle+\left\langle\varphi, b^{-1} d b\right\rangle .
$$

The second term is closed and can only contribute when $G_{\varphi}$ has a non-trivial topology. At the classical level, we can ignore this term. Therefore, we see that the action depends only on $G / G_{\varphi} \simeq \mathcal{O}_{\varphi}$. In order to get an explicit expression of the Lagrangian for a given $G$ and $\varphi$, it is sufficient to take an appropriate parametrisation of $G / G_{\varphi} \simeq \mathcal{O}_{\varphi}$. For instance, in the Poincaré case with $\varphi=m \mathcal{P}^{0}$, we can use

$$
g=\exp \left(x^{a} P_{a}\right) \exp \left(v^{i} M_{0 i}\right) R,
$$

where $R$ is a rotation element in $\mathrm{SO}(D-1)$, and we can drop it as it contributes to the action only as a boundary term. We can also remove $e^{x^{0} P_{0}}$ using

$$
\exp \left(v^{i} M_{0 i}\right) e^{\tau P_{0}}=\exp \left(\tau\left(\cosh v P_{0}+\frac{\sinh v}{v} v^{i} P_{i}\right)\right) \exp \left(v^{i} M_{0 i}\right)
$$

With a choice of $\tau \cosh v=x^{0}$, we find

$$
g=\exp \left(\tilde{x}^{i} P_{i}\right) \exp \left(v^{i} M_{0 i}\right) \exp \left(-\frac{x^{0}}{\cosh v} P_{0}\right) R,
$$

with

$$
\tilde{x}^{i}=x^{i}-x^{0} \frac{\tanh v}{v} v^{i} .
$$

Discarding the last two factors in (C.9) since they belong to the stabiliser, we obtain the action,

$$
\begin{aligned}
S & =m \int d t\left\langle\mathcal{P}^{0}, \exp \left(-v^{i} M_{0 i}\right) \dot{\tilde{x}}^{i} P_{i} \exp \left(v^{i} M_{0 i}\right)\right\rangle \\
& =-m \int d t \frac{\sinh v}{v} v_{i} \dot{\tilde{x}}^{i} .
\end{aligned}
$$

If we redefine $v^{i}$ as

$$
\tilde{p}_{i}=-m \frac{\sinh v}{v} v_{i},
$$

we end up with an action without Hamiltonian,

$$
S=\int d t \tilde{p}_{i} \dot{\tilde{x}}^{i} .
$$

If we had not done the reordering of factors in $g$, we would have obtained

$$
S=m \int d t\left(\cosh v \dot{x}^{0}-\frac{\sinh v}{v} v_{i} \dot{x}^{i}\right) .
$$

One can also derive the above form of the action by plugging (C.10) into (C.11) and performing an integration by part. Since the solution of (C.11) is simply,

$$
\tilde{x}^{i}=\text { const }, \quad v^{i}=\text { const },
$$

the relation (C.10) provides the general solution of the ordinary relativistic massive particle system (C.14). 
The fact that we can bring the action (C.14) to the form (C.13) is not surprising because the starting point of the action was the pullback of the Liouville one-form on the orbit. Locally, we can always choose Darboux coordinates, then the action will take the form of (C.13). From this perspective, the dynamics of the system is inherited from the coordinate choice for the orbit, or equivalently the group element $g$. In order to make certain symmetries of an orbit manifest it is useful to choose appropriate coordinates on the orbit. Moreover, global properties of the orbit have to be taken into account.

\section{From the coloured Hamiltonian to the Lagrangian action}

In the uncoloured case, we can obtain a quadratic action by integrating out the momenta $p_{a}$. We can try the same for the coloured particle (4.26) where the equation for momenta becomes matrix equations

$$
\dot{\mathbb{X}}+\mathbb{P} \mathbb{L}+\mathbb{L} \mathbb{P}=0 .
$$

In order to solve this equation, we assume the invertibility of $\mathbb{L}$ and deform the equation by introducing $\epsilon$ as

$$
\mathbb{P}=-\mathbb{L}^{-1} \dot{\boldsymbol{X}}-\epsilon \mathbb{L}^{-1} \mathbb{P} \mathbb{L} .
$$

Eventually, we will take $\epsilon \rightarrow 1$ limit, but in the intermediate step we will keep $\epsilon$ which plays the role of expansion parameter and regulator. The solution of the above as a power seires in $\epsilon$ is

$$
\mathbb{P}=-\sum_{n=0}^{\infty}(-\epsilon)^{n} \mathbb{L}^{-n-1} \dot{\mathfrak{X}} \mathbb{L}^{n}
$$

The Lagrangian (4.26) becomes

$$
\begin{aligned}
2 N L= & -\sum_{n=0}^{\infty}(-\epsilon)^{n} \operatorname{Tr}\left(\mathbb{L}^{-n-1} \dot{\mathbf{X}} \mathbb{L}^{n} \dot{\mathbf{X}}\right) \\
& +\sum_{m=0}^{\infty} \sum_{n=0}^{\infty}(-\epsilon)^{m+n} \operatorname{Tr}\left(\mathbb{L}^{-n+m} \dot{\mathbf{X}} \mathbb{L}^{n-m-1} \dot{\mathbf{X}}\right)+m^{2} \operatorname{Tr}(\mathbb{L}) .
\end{aligned}
$$

We can perform a resummation for the double sum in the second line as

$$
\sum_{m=0}^{\infty} \sum_{n=0}^{\infty}(-\epsilon)^{m+n} \operatorname{Tr}\left(\mathbb{L}^{-n+m} \dot{\mathbf{X}} \mathbb{L}^{n-m-1} \dot{\boldsymbol{X}}\right)=\frac{1}{1+\epsilon} \sum_{n=0}^{\infty}(-\epsilon)^{n} \operatorname{Tr}\left(\mathbb{L}^{-n-1} \dot{\boldsymbol{X}} \mathbb{L}^{n} \dot{\mathbf{X}}\right) .
$$

Above, we split the double sum into two parts, $m \geq n$ and $n>m$, and each parts gives the prefactors $\frac{1}{1-\epsilon^{2}}$ and $\frac{-\epsilon}{1-\epsilon^{2}}$ respectively, hence it is important to keep $|\epsilon|<1$ for the convergence of these terms. Note however that the final result (the sum of two prefactors) is regular in $\epsilon$. Using the above solution, we find the quadratic action as

$$
2 N L=-\frac{\epsilon}{1+\epsilon} \sum_{n=0}^{\infty}(-\epsilon)^{n} \operatorname{Tr}\left(\mathbb{L}^{-n-1} \dot{\boldsymbol{X}} \mathbb{L}^{n} \dot{\boldsymbol{X}}\right)+m^{2} \operatorname{Tr}(\mathbb{L}) .
$$

As mentioned above, the above series do not exhibit any apparent divergence in the $\epsilon \rightarrow 1$ limit, but we do not know whether the series converges. 
For a more rigorous treatment, let us assume that the matrix $\mathbb{L}$ can be diagonalised as

$$
\mathbb{L}=\mathbb{S} \operatorname{diag}\left(\lambda_{1}, \ldots, \lambda_{2 N}\right) \mathbb{S}^{-1}, \quad\left|\lambda_{1}\right|>\left|\lambda_{2}\right|>\cdots>\left|\lambda_{2 N}\right|>0 .
$$

Then $\mathbb{P}$ can be solved as

$$
\left(\mathbb{S}^{-1} \mathbb{P} \mathbb{S}\right)_{i j}=-\frac{1}{\lambda_{i}+\lambda_{j}}\left(\mathbb{S}^{-1} \dot{\boldsymbol{X}} \mathbb{S}\right)_{i j}
$$

and the Lagrangian reads

$$
2 N L=-\frac{1}{2} \sum_{i, j=1}^{2 N} \frac{1}{\lambda_{i}+\lambda_{j}}\left(\mathbb{S}^{-1} \dot{\boldsymbol{\aleph}} \mathbb{S}\right)_{i j}\left(\mathbb{S}^{-1} \dot{\boldsymbol{K}} \mathbb{S}\right)_{j i}+m^{2} \sum_{i=1}^{2 N} \lambda_{i}
$$

Since the factor $\left(\lambda_{i}+\lambda_{j}\right)^{-1}$ prevents us to re-express the above as a trace, we can try to expand it so that each term in the expansion could be written as a trace. However, for the convergence of the expansion we need to distinguish cases $i>j, i<j$ and $i=j$, and we can obtain at best

$2 N L=-\sum_{n=0}^{\infty}(-1)^{n} \sum_{i>j} \lambda_{i}^{-n-1}\left(\mathbb{S}^{-1} \dot{\boldsymbol{\aleph}} \mathbb{S}\right)_{i j} \lambda_{j}^{n}\left(\mathbb{S}^{-1} \dot{\boldsymbol{\aleph}} \mathbb{S}\right)_{j i}-\frac{1}{2} \sum_{i} \lambda_{i}^{-1}\left(\mathbb{S}^{-1} \dot{\boldsymbol{\aleph}} \mathbb{S}\right)_{i i}^{2}+m^{2} \sum_{i=1}^{2 N} \lambda_{i}$.

If there were no restriction $i>j$, we could express the first series as

$$
-\sum_{n=0}^{\infty}(-1)^{n} \sum_{i, j} \lambda_{i}^{-n-1}\left(\mathbb{S}^{-1} \dot{\boldsymbol{\chi}} \mathbb{S}\right)_{i j} \lambda_{j}^{n}\left(\mathbb{S}^{-1} \dot{\boldsymbol{\chi}} \mathbb{S}\right)_{j i}=-\sum_{n=0}^{\infty}(-1)^{n} \operatorname{Tr}\left(\mathbb{L}^{-n-1} \dot{\boldsymbol{k}} \mathbb{L}^{n} \dot{\boldsymbol{k}}\right),
$$

but because of this restriction we cannot. Since the restricted sum $\sum_{i>j}$ can be understood as "one half" of the unrestricted sum $\sum_{i, j}$, one may have an intuitive understanding of the expression (D.5).

Now, let us integrate out the Lagrange multiplier. We can do it again in the diagonalised case. The equation for the eigenvalue $\lambda_{i}$ is

$$
\sum_{j=1}^{2 N} \frac{1}{\left(\lambda_{i}+\lambda_{j}\right)^{2}} A_{i j}+m^{2}=0, \quad i=1, \ldots, 2 N
$$

where we defined

$$
A_{i j}=A_{j i}=\left(\mathbb{S}^{-1} \dot{\mathbf{X}} \mathbb{S}\right)_{i j}\left(\mathbb{S}^{-1} \dot{\boldsymbol{\chi}} \mathbb{S}\right)_{j i}
$$

For $N=1$, we find

$$
\frac{1}{4 \lambda_{1}^{2}} A_{11}+\frac{1}{\left(\lambda_{1}+\lambda_{2}\right)^{2}} A_{12}=-m^{2}, \quad \frac{1}{4 \lambda_{2}^{2}} A_{22}+\frac{1}{\left(\lambda_{1}+\lambda_{2}\right)^{2}} A_{12}=-m^{2} .
$$

The equations can be re-expressed as a quartic one so they have four different solutions. Since the Lagrangian is proportional to the trace $\operatorname{Tr}(\mathbb{L})=\lambda_{1}+\lambda_{2}$, we can focus on it and find

$$
\begin{aligned}
-4 m^{2}\left(\lambda_{1}+\lambda_{2}\right)^{2} & =4 A_{12}+\left(\sqrt{A_{11}} \pm \sqrt{A_{22}}\right)^{2} \\
& =2\left(A_{11}+2 A_{12}+A_{22}\right)-\left(\sqrt{A_{11}} \mp \sqrt{A_{22}}\right)^{2} \\
& =2 \operatorname{Tr}\left(\dot{\boldsymbol{X}}^{2}\right)-\left(\left(\mathbb{S}^{-1} \dot{\boldsymbol{X}} \mathbb{S}\right)_{11} \mp\left(\mathbb{S}^{-1} \dot{\boldsymbol{X}} \mathbb{S}\right)_{22}\right)^{2} .
\end{aligned}
$$


When the relative sign in the last line is postive then it can be also expressed as a trace (which is zero in this case), and we find

$$
-4 m^{2}\left(\lambda_{1}+\lambda_{2}\right)^{2}=2 \operatorname{Tr}\left(\dot{\boldsymbol{X}}^{2}\right)-(\operatorname{Tr}(\dot{\mathcal{X}}))^{2}=2 \operatorname{Tr}\left(\dot{\boldsymbol{X}}^{2}\right) .
$$

For $N=2$, we find a set of equations equivalent to a single order-eight one, and hence would contain eight solutions.

Open Access. This article is distributed under the terms of the Creative Commons Attribution License (CC-BY 4.0), which permits any use, distribution and reproduction in any medium, provided the original author(s) and source are credited.

\section{References}

[1] S.R. Coleman and J. Mandula, All Possible Symmetries of the S Matrix, Phys. Rev. 159 (1967) 1251 [INSPIRE].

[2] A. Achucarro and P.K. Townsend, A Chern-Simons Action for Three-Dimensional anti-de Sitter Supergravity Theories, Phys. Lett. B 180 (1986) 89 [InSPIRE].

[3] E. Witten, (2+1)-Dimensional Gravity as an Exactly Soluble System, Nucl. Phys. B 311 (1988) 46 [INSPIRE].

[4] M.P. Blencowe, A Consistent Interacting Massless Higher Spin Field Theory in D $=(2+1)$, Class. Quant. Grav. 6 (1989) 443 [inSPIRE].

[5] A. Campoleoni, S. Fredenhagen, S. Pfenninger and S. Theisen, Asymptotic symmetries of three-dimensional gravity coupled to higher-spin fields, JHEP 11 (2010) 007 [arXiv: 1008.4744] [INSPIRE].

[6] M. Grigoriev, K. Mkrtchyan and E. Skvortsov, Matter-free higher spin gravities in 3D: Partially-massless fields and general structure, Phys. Rev. D 102 (2020) 066003 [arXiv: 2005.05931] [INSPIRE].

[7] P. Salgado, R.J. Szabo and O. Valdivia, Topological gravity and transgression holography, Phys. Rev. D 89 (2014) 084077 [arXiv:1401.3653] [InSPIRE].

[8] S. Hoseinzadeh and A. Rezaei-Aghdam, (2+1)-dimensional gravity from Maxwell and semisimple extension of the Poincaré gauge symmetric models, Phys. Rev. D 90 (2014) 084008 [arXiv: 1402.0320] [INSPIRE].

[9] G. Papageorgiou and B.J. Schroers, Galilean quantum gravity with cosmological constant and the extended q-Heisenberg algebra, JHEP 11 (2010) 020 [arXiv: 1008. 0279] [INSPIRE].

[10] E.A. Bergshoeff and J. Rosseel, Three-Dimensional Extended Bargmann Supergravity, Phys. Rev. Lett. 116 (2016) 251601 [arXiv: 1604.08042] [INSPIRE].

[11] J. Hartong, Y. Lei and N.A. Obers, Nonrelativistic Chern-Simons theories and three-dimensional Hořava-Lifshitz gravity, Phys. Rev. D 94 (2016) 065027 [arXiv: 1604.08054] [inSPIRE].

[12] L. Avilés, E. Frodden, J. Gomis, D. Hidalgo and J. Zanelli, Non-Relativistic Maxwell Chern-Simons Gravity, JHEP 05 (2018) 047 [arXiv: 1802. 08453] [INSPIRE].

[13] S. Gwak, E. Joung, K. Mkrtchyan and S.-J. Rey, Rainbow Valley of Colored (Anti) de Sitter Gravity in Three Dimensions, JHEP 04 (2016) 055 [arXiv: 1511.05220] [INSPIRE]. 
[14] S. Gwak, E. Joung, K. Mkrtchyan and S.-J. Rey, Rainbow vacua of colored higher-spin (A) $d S_{3}$ gravity, JHEP 05 (2016) 150 [arXiv:1511.05975] [INSPIRE].

[15] S.E. Konstein and M.A. Vasiliev, Extended Higher Spin Superalgebras and Their Massless Representations, Nucl. Phys. B 331 (1990) 475 [InSPIRE].

[16] R.M. Wald, Spin-2 Fields and General Covariance, Phys. Rev. D 33 (1986) 3613 [InSPIRE].

[17] C. Cutler and R.M. Wald, A New Type of Gauge Invariance for a Collection of Massless Spin-2 Fields. 1. Existence and Uniqueness, Class. Quant. Grav. 4 (1987) 1267 [INSPIRE].

[18] R.M. Wald, A New Type of Gauge Invariance for a Collection of Massless Spin-2 Fields. 2. Geometrical Interpretation, Class. Quant. Grav. 4 (1987) 1279 [InSPIRE].

[19] N. Boulanger, T. Damour, L. Gualtieri and M. Henneaux, Inconsistency of interacting, multigraviton theories, Nucl. Phys. B 597 (2001) 127 [hep-th/0007220] [InSPIRE].

[20] N. Boulanger and L. Gualtieri, An exotic theory of massless spin two fields in three-dimensions, Class. Quant. Grav. 18 (2001) 1485 [hep-th/0012003] [INSPIRE].

[21] E. Joung and W. Li, Nonrelativistic limits of colored gravity in three dimensions, Phys. Rev. D 97 (2018) 105020 [arXiv: 1801.10143] [INSPIRE].

[22] S.F. Prokushkin and M.A. Vasiliev, Higher spin gauge interactions for massive matter fields in 3-D AdS space-time, Nucl. Phys. B 545 (1999) 385 [hep-th/9806236] [INSPIRE].

[23] S. Prokushkin and M.A. Vasiliev, 3-D higher spin gauge theories with matter, hep-th/9812242 [INSPIRE].

[24] R. Bonezzi, N. Boulanger, E. Sezgin and P. Sundell, An Action for Matter Coupled Higher Spin Gravity in Three Dimensions, JHEP 05 (2016) 003 [arXiv: 1512.02209] [INSPIRE].

[25] P.O. Kazinski, S.L. Lyakhovich and A.A. Sharapov, Lagrange structure and quantization, JHEP 07 (2005) 076 [hep-th/0506093] [INSPIRE].

[26] S. Fredenhagen, O. Krüger and K. Mkrtchyan, Vertex-Constraints in 3D Higher Spin Theories, Phys. Rev. Lett. 123 (2019) 131601 [arXiv:1905.00093] [INSPIRE].

[27] D.A. Vogan, Gelfand-Kirillov dimension for Harish-Chandra module, Invent. Math. 48 (1978) 75 .

[28] H. Bacry, P. Combe and J.L. Richard, Group-theoretical analysis of elementary particles in an external electromagnetic field. 1. the relativistic particle in a constant and uniform field, Nuovo Cim. A 67 (1970) 267 [INSPIRE].

[29] R. Schrader, The maxwell group and the quantum theory of particles in classical homogeneous electromagnetic fields, Fortsch. Phys. 20 (1972) 701 [INSPIRE].

[30] S. Bonanos and J. Gomis, Infinite Sequence of Poincaré Group Extensions: Structure and Dynamics, J. Phys. A 43 (2010) 015201 [arXiv:0812.4140] [InSPIRE].

[31] J. Gomis and A. Kleinschmidt, On free Lie algebras and particles in electro-magnetic fields, JHEP 07 (2017) 085 [arXiv:1705.05854] [INSPIRE].

[32] S.K. Wong, Field and particle equations for the classical Yang-Mills field and particles with isotopic spin, Nuovo Cim. A 65 (1970) 689 [INSPIRE].

[33] M.R. Brown and M.J. Duff, Exact Results for Effective Lagrangians, Phys. Rev. D 11 (1975) 2124 [INSPIRE].

[34] I.A. Batalin, S.G. Matinyan and G.K. Savvidy, Vacuum Polarization by a Source-Free Gauge Field, Sov. J. Nucl. Phys. 26 (1977) 214 [inSPIRE]. 
[35] J.-M. Souriau, Structure des systèmes dynamiques, Dunod (1970), English translation: Structure of Dynamical Systems: A Symplectic View of Physics, Birkhäuser (1997), [DOI].

[36] V. de Alfaro, S. Fubini and G. Furlan, Conformal Invariance in Quantum Mechanics, Nuovo Cim. A 34 (1976) 569 [INSPIRE].

[37] J. Fuchs and C. Schweigert, Symmetries, Lie algebras and representations: A graduate course for physicists, Cambridge University Press (2003).

[38] M. Hatsuda and M. Sakaguchi, Wess-Zumino term for the AdS superstring and generalized Inonu-Wigner contraction, Prog. Theor. Phys. 109 (2003) 853 [hep-th/0106114] [InSPIRE].

[39] N. Boulanger, M. Henneaux and P. van Nieuwenhuizen, Conformal (super)gravities with several gravitons, JHEP 01 (2002) 035 [hep-th/0201023] [INSPIRE].

[40] J.A. de Azcarraga, J.M. Izquierdo, M. Picón and O. Varela, Generating Lie and gauge free differential (super)algebras by expanding Maurer-Cartan forms and Chern-Simons supergravity, Nucl. Phys. B 662 (2003) 185 [hep-th/0212347] [INSPIRE].

[41] F. Izaurieta, E. Rodriguez and P. Salgado, Expanding Lie (super)algebras through Abelian semigroups, J. Math. Phys. 47 (2006) 123512 [hep-th/0606215] [INSPIRE].

[42] J. Matulich, S. Prohazka and J. Salzer, Limits of three-dimensional gravity and metric kinematical Lie algebras in any dimension, JHEP 07 (2019) 118 [arXiv:1903.09165] [INSPIRE].

[43] A. Barducci, R. Casalbuoni and J. Gomis, Nonrelativistic k-contractions of the coadjoint Poincaré algebra, Int. J. Mod. Phys. A 35 (2020) 2050009 [arXiv:1910.11682] [InSPIRE].

[44] T. Fukuyama and K. Kamimura, Gauge Theory of Two-dimensional Gravity, Phys. Lett. B 160 (1985) 259 [INSPIRE].

[45] K. Isler and C.A. Trugenberger, A Gauge Theory of Two-dimensional Quantum Gravity, Phys. Rev. Lett. 63 (1989) 834 [INSPIRE].

[46] A.H. Chamseddine and D. Wyler, Topological Gravity in (1+1)-dimensions, Nucl. Phys. B 340 (1990) 595 [INSPIRE].

[47] K.B. Alkalaev, On higher spin extension of the Jackiw-Teitelboim gravity model, J. Phys. A 47 (2014) 365401 [arXiv: 1311.5119] [INSPIRE].

[48] K. Alkalaev, E. Joung and J. Yoon, in preparation.

[49] E. Bergshoeff, W. Merbis, A.J. Routh and P.K. Townsend, The Third Way to 3D Gravity, Int. J. Mod. Phys. D 24 (2015) 1544015 [arXiv:1506.05949] [InSPIRE].

[50] S. Deser, R. Jackiw and S. Templeton, Topologically Massive Gauge Theories, Annals Phys. 140 (1982) 372 [Erratum ibid. 185 (1988) 406] [INSPIRE].

[51] A.S. Schwarz, The Partition Function of Degenerate Quadratic Functional and Ray-Singer Invariants, Lett. Math. Phys. 2 (1978) 247 [InSPIRE].

[52] G.T. Horowitz, Exactly Soluble Diffeomorphism Invariant Theories, Commun. Math. Phys. 125 (1989) 417 [INSPIRE].

[53] A.S. Cattaneo and C.A. Rossi, Higher dimensional BF theories in the Batalin-Vilkovisky formalism: the BV action and generalized Wilson loops, Commun. Math. Phys. 221 (2001) 591 [math/0010172] [INSPIRE].

[54] S.R. Coleman, J. Wess and B. Zumino, Structure of phenomenological Lagrangians. 1, Phys. Rev. 177 (1969) 2239 [INSPIRE]. 
[55] C.G. Callan Jr., S.R. Coleman, J. Wess and B. Zumino, Structure of phenomenological Lagrangians. 2, Phys. Rev. 177 (1969) 2247 [INSPIRE].

[56] V.I. Ogievetsky, Non-linear realizations of internal and spacetime symmetries, in Proc. 10th Karpacz Winter School of Theoretical physics (1974).

[57] J. Gomis, K. Kamimura and P.C. West, The construction of brane and superbrane actions using non-linear realisations, Class. Quant. Grav. 23 (2006) 7369 [hep-th/0607057] [INSPIRE].

[58] D.H. Collingwood and W.M. McGovern, Nilpotent orbits in semisimple Lie algebras, Van Nostrand Reinhold Mathematics Series. Van Nostrand Reinhold Co., New York, U.S.A. (1993), [DOI].

[59] A. Barducci, R. Casalbuoni and L. Lusanna, Classical Scalar and Spinning Particles Interacting with External Yang-Mills Fields, Nucl. Phys. B 124 (1977) 93 [inSPIRE].

[60] J. Gomis, A. Kleinschmidt, D. Roest and P. Salgado-ReboLledó, A free Lie algebra approach to curvature corrections to flat space-time, JHEP 09 (2020) 068 [arXiv:2006.11102] [INSPIRE].

[61] P. Salgado and S. Salgado, $\mathfrak{s o}(D-1,1) \otimes \mathfrak{s o}(D-1,2)$ algebras and gravity, Phys. Lett. $B$ 728 (2014) 5 [INSPIRE].

[62] J. Gomis, A. Kleinschmidt and J. Palmkvist, Galilean free Lie algebras, JHEP 09 (2019) 109 [arXiv: 1907.00410] [INSPIRE].

[63] J. Brugues, T. Curtright, J. Gomis and L. Mezincescu, Non-relativistic strings and branes as non-linear realizations of Galilei groups, Phys. Lett. B 594 (2004) 227 [hep-th/0404175] [INSPIRE].

[64] M. Kontsevich, Deformation quantization of Poisson manifolds, I, q-alg/9709040.

[65] M.A. Vasiliev, Relativity, causality, locality, quantization and duality in the $S(p)(2 M)$ invariant generalized space-time, hep-th/0111119 [INSPIRE].

[66] D. Sorokin and M. Tsulaia, Higher Spin Fields in Hyperspace. A Review, Universe 4 (2018) 7 [arXiv: 1710.08244] [INSPIRE].

[67] E. Joung and K. Mkrtchyan, Notes on higher-spin algebras: minimal representations and structure constants, JHEP 05 (2014) 103 [arXiv:1401.7977] [INSPIRE].

[68] T. Basile, E. Joung, K. Mkrtchyan and M. Mojaza, Dual Pair Correspondence in Physics: Oscillator Realizations and Representations, JHEP 09 (2020) 020 [arXiv:2006.07102] [INSPIRE].

[69] E. Joung and K. Mkrtchyan, Partially-massless higher-spin algebras and their finite-dimensional truncations, JHEP 01 (2016) 003 [arXiv: 1508.07332] [INSPIRE].

[70] J.F. Schonfeld, A Mass Term for Three-Dimensional Gauge Fields, Nucl. Phys. B 185 (1981) 157.

[71] M.S. Plyushchay, The model of relativistic particle with torsion, Nucl. Phys. B 362 (1991) 54 [INSPIRE].

[72] L. Mezincescu and P.K. Townsend, Semionic Supersymmetric Solitons, J. Phys. A 43 (2010) 465401 [arXiv: 1008.2775 ] [INSPIRE].

[73] C. Batlle, J. Gomis, K. Kamimura and J. Zanelli, Dynamical sectors for a spinning particle in $A d S_{3}$, Phys. Rev. D 90 (2014) 065017 [arXiv:1407.2355] [INSPIRE]. 